\title{
Cryo-EM Structures of CTP Synthase Filaments Reveal Mechanism of pH-Sensitive Assembly During Budding Yeast Starvation
}

Jesse M Hansen ${ }^{1,2 \#}$, Avital Horowitz ${ }^{1 \#}$, Eric M Lynch ${ }^{1}$, Daniel P Farrell ${ }^{1}$, Joel Quispe ${ }^{1}$, Frank DiMaio $^{1}$, Justin M Kollman ${ }^{1 *}$

\#These authors contributed equally.

1 Department of Biochemistry, University of Washington, Seattle, USA

2 Graduate Program in Biological Physics, Structure, and Design, University of Washington, Seattle, USA

${ }^{*}$ Corresponding author. Email: jkoll@uw.edu

\begin{abstract}
Many metabolic enzymes self-assemble into micron-scale filaments to organize and regulate metabolism. The appearance of these assemblies often coincides with large metabolic changes as in development, cancer, and stress. Yeast undergo cytoplasmic acidification upon starvation, triggering the assembly of many metabolic enzymes into filaments. However, it is unclear how these filaments assemble at the molecular level and what their role is in the yeast starvation response. CTP Synthase (CTPS) assembles into metabolic filaments across many species. Here, we characterize in vitro polymerization and investigate in vivo consequences of CTPS assembly in yeast. Cryo-EM structures reveal a $\mathrm{pH}$-sensitive assembly mechanism and highly ordered filament bundles that stabilize an inactive state of the enzyme, features unique to yeast CTPS. Disruption of filaments in cells with non-assembly or hyper-assembly mutations decreases growth rate, reflecting the importance of regulated CTPS filament assembly in homeotstasis.
\end{abstract}

\section{INTRODUCTION}

Intermediate metabolism is finely tuned, carefully balanced, and robustly adaptable to changes in environmental conditions. More than a century of effort has gone into understanding the connections between pathways, the individual enzymes that drive the biochemistry, and the regulation of metabolic flux. Only recently, however, have we come to appreciate the role of metabolic enzyme self-assembly as a widespread mechanism of metabolic organization and regulation (Lynch et al., 2020; Park and Horton, 2019; Simonet et al., 2020). These assemblies have been found in many core pathways including glycolysis (Kemp, 1971; Webb et al., 2017), fatty acid synthesis (Hunkeler et al., 2018; Kleinschmidt et al., 1969), amino acid synthesis (Cohen et al., 1976; Frey et al., 1975; Miller et al., 1974; Petrovska et al., 2014; Zhang et al., 2018), and nucleotide synthesis (Barry et al., 2014; Carcamo et al., 2011). Metabolic enzymes that form filaments are frequently found at rate-limiting and energetically-committed steps of pathways, suggesting filaments play a role in regulating metabolic flux (Noree et al., 2019). Indeed, for most enzyme polymers that have been functionally characterized, assembly functions as a mechanism of allosteric regulation to tune enzyme activity (Barry et al., 2014; Hunkeler et al., 2018; Johnson and Kollman, 2020; Lynch et al., 2017; Lynch and Kollman, 2020; Stoddard et al., 2020).

CTP synthase (CTPS) catalyzes the final, rate-limiting step of de novo CTP biosynthesis (Lieberman, 1956) (Fig 1a). Its biochemistry and structure have been studied extensively, making it an ideal model enzyme for exploring the role of enzyme assembly in regulating activity. Each CTPS monomer consists of a glutaminase domain and an amido-ligase domain 
connected by an alpha helical linker (Fig 1b). Glutamine hydrolysis in the glutaminase domain produces ammonia which is transferred through a channel into the amido-ligase domain, where it is ligated to UTP to form CTP in an ATP-dependent process (Endrizzi et al., 2005; Goto et al., 2004). Substrate binding induces rotation of the glutaminase domain which opens an ammonia channel between active sites (Lynch and Kollman, 2020), and it's reaction product CTP inhibits activity (Habrian et al., 2016). CTPS assembles X-shaped, D2 symmetric homotetramers through interactions of the amido-ligase domains, with multiple protomers participating in each substrate and regulatory binding site (Fig 1c).

Cellular CTPS filament assembly is widespread across the domains of life, having been observed in bacteria, archaea, and eukaryotes (Carcamo et al., 2011; Ingerson-Mahar et al., 2010; Liu, 2010; Noree et al., 2010; Zhou et al., 2021). We have previously shown, however, that there are striking differences between the assembly mechanisms and filament architectures among species (Fig 1d-f). These differences give rise to differences in function, with bacterial CTPS filaments providing a mechanism to allosterically inhibit the enzyme, while animal CTPS filaments act to increase activity and cooperativity of regulation (Barry et al., 2014;Lynch et al., 2017; Lynch and Kollman, 2020; Zhou et al., 2021, 2019).

In budding yeast, regulation of metabolism is key to survival as sudden fluctuations in environmental resources is common. During starvation, yeast assemble many metabolic enzymes into filaments (Narayanaswamy et al., 2009; Noree et al., 2010; Shen et al., 2016) which protects cells and permits rapid growth upon readdition of nutrients (Petrovska et al., 2014). It is thought that polymerization leads to increased cytoplasmic viscosity, limiting diffusion of metabolites and slowing growth (Petrovska et al., 2014). Without energy, membrane-bound proton pumps fail and the neutral-pH cytoplasm is acidified (Orij et al., 2009). This acidification is necessary and sufficient for yeast to trigger metabolic filament assembly and effectively mount a stress response (Petrovska et al., 2014). Therefore, yeast present a unique circumstance to study metabolic filament assembly as it relates to the stress response. Fluorescence-based studies of tagged CTPS in yeast and other organisms demonstrated large foci or rods consistent with laterally associating filaments (Ingerson-Mahar et al., 2010; Liu, 2010), yet there are no high resolution structures of bundled metabolic enzymes in any organism. The molecular basis of starvation-driven assembly remains unknown as well as whether polymer assembly regulates enzymatic activity of CTPS during the yeast stress response.

Budding yeast have two CTPS isoforms, Ura7 (Yang et al., 1994) and Ura8 (Nadkarni et al., 1995; Ozier-Kalogeropoulos et al., 1994), which share $78 \%$ identical residues. While Ura7 mRNA is 2-fold more abundant than Ura8 in vivo (Nadkarni et al., 1995), they are functionally overlapping and deletion of either gene results in slowed growth (Ozier-Kalogeropoulos et al., 1994). Both form supramolecular structures in response to stress that colocalize in cells (Noree et al., 2010; Shen et al., 2016).

Here, we investigate the structure and in vivo function of yeast CTPS filaments. We use cryoelectron microscopy (cryo-EM) to determine the molecular mechanism of yeast CTPS filament assembly and higher-order bundled assemblies. The interface between tetramers in the yeast CTPS filament, which is unique among the existing CTPS filament structures, explains the $\mathrm{pH}$ sensitivity of assembly. Two engineered mutations at this assembly interface, one that disrupts filaments and one that stabilizes them, allow us to probe the functional role of filament assembly 
in vitro and in vivo. Yeast CTPS filaments stabilized a conformation that pinches shut the ammonia channel between catalytic sites, which reduces activity in the filament. Both nonassembling and hyper-assembling CTPS mutants lead to slowed proliferation, indicating the critical role of regulated CTPS assembly in both vegetative growth and recovery from starvation.
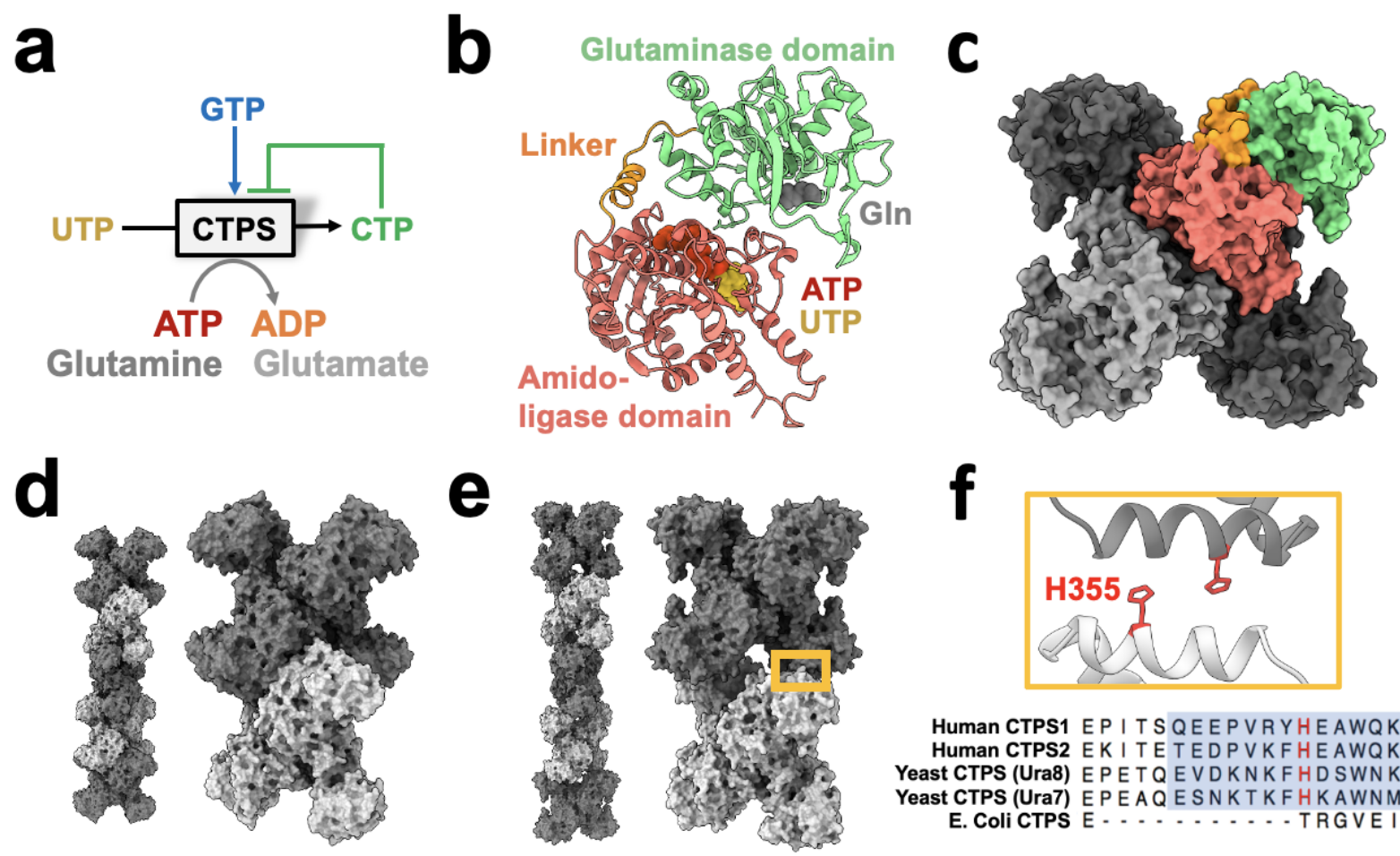

Human CTPS1 EPITSQEEPVRYHEAWQKL Human CTPS2 EK I TETEDPVKFHEAWQKL Yeast CTPS (Ura8) EPETQEVDKNKFHDSWNKL Yeast CTPS (Ura7) EPEAQESNKTKFHKAWNMV E. Coli CTPS E............ TRG VE I L

Figure 1: CTPS canonical structure and filament assembly. (a) De novo CTP synthesis reaction diagram. (b) Substrate-bound hCTPS2 (6PK4) monomer with glutamine modelled from 1VCO. (c) Canonical tetramer structure of CTPS (6PK4). Monomers are shaded differently, and domain colors from panel B are painted for one monomer. (d) Surface model display for filament assembly of stacked tetramers for product-bound E. coli CTPS (5U3C). (e) Surface model display for filament assembly of stacked tetramers for product-bound hCTPS2 (6PK7). (f) Zoom-in of box from panel $\mathrm{E}$ showing filament assembly interface, with conserved $\mathrm{H} 355$ in red. Below, sequence alignment of filament assembly interface, with blue shaded box for eukaryotic alpha helical insert and conserved key histidine in red.

\section{RESULTS}

\subsection{Yeast CTPS filament assembly is pH-sensitive}

It remains an open question whether $\mathrm{pH}$-regulated assembly is an inherent feature of budding yeast CTPS, or whether other cellular factors are required for assembly. To address this, we first confirmed prior work that showed yeast CTPS assembles in cells upon cytoplasmic acidification (Petrovska et al., 2014); Ura7-GFP tagged at the endogenous locus forms foci upon nutrient deprivation (Fig 2a), or when cells are permeabilized with DNT to manipulate cytoplasmic $\mathrm{pH}$ (Fig 2b). We next examined polymerization of purified recombinant Ura7 and Ura8 at different $\mathrm{pHs}$ by negative stain EM. Apo CTPS did not form polymers at any $\mathrm{pH}$. At $\mathrm{pH}$ 7.4 both isoforms assembled short, single filaments on binding substrates or product, but pH 6.0 
promoted assembly of much larger polymers that appeared to be bundled filaments (Fig 2c; Fig2 Supp 1). Thus, like other species (Barry et al., 2014; Lynch et al., 2017; Zhou et al., 2019), yeast CTPS assembly is dependent on ligand binding, and the $\mathrm{pH}$ sensitivity is intrinsic to the enzyme itself.

We wondered whether the intrinsic $\mathrm{pH}$ sensitivity of yeast CTPS was conserved among other species. Previous studies have not described the $\mathrm{pH}$-sensitivity of human CTPS filament assembly, and have shown robust assembly at pH 8.0 (Lynch et al., 2017; Lynch and Kollman, 2020). The overall sequence conservation between human and yeast CTPS, and the presence of a histidine residue at the assembly interface in human CTPS filaments, led us to consider whether human CTPS assembly is also $\mathrm{pH}$-dependent (Fig 1d). We observed equally robust polymerization of purified human CTPS2 (hCTPS2) at pH 6.0 and 8.0, and negative stain EM averages show that filaments formed at different $\mathrm{pHs}$ have similar architectures, suggesting there is no direct effect of pH on hCTPS2 assembly or structure (Fig. 2d, inserts). Thus, pHdriven assembly of CTPS appears to be specific to yeast, raising the question of what unique structural features might confer $\mathrm{pH}$-sensitivity.

To assess the kinetics of $\mathrm{pH}$-dependent CTPS assembly, we monitored right angle light scattering by CTPS after addition of CTP (Fig 2e). Both isoforms had very low signal at $\mathrm{pH} 7$, consistent with our negative stain imaging. At pH 6, Ura8 assembly was much faster than Ura7, but inspection at early time points showed that both exhibited biphasic assembly which could not be fit with a single equation (Fig2 Supp 2). Instead, early and late assembly kinetics fit well to separate four-parameter curves, indicating potentially distinct assembly phenomena with different kinetics in the early and late phases. We examined the growth of Ura7 filaments by negative stain EM over time, and found that single filaments appear at early time points and the thicker bundles at later time points, suggesting that the biphasic scattering kinetics can be explained by initial linear polymerization followed by lateral aggregation (Fig2 Supp 2). 

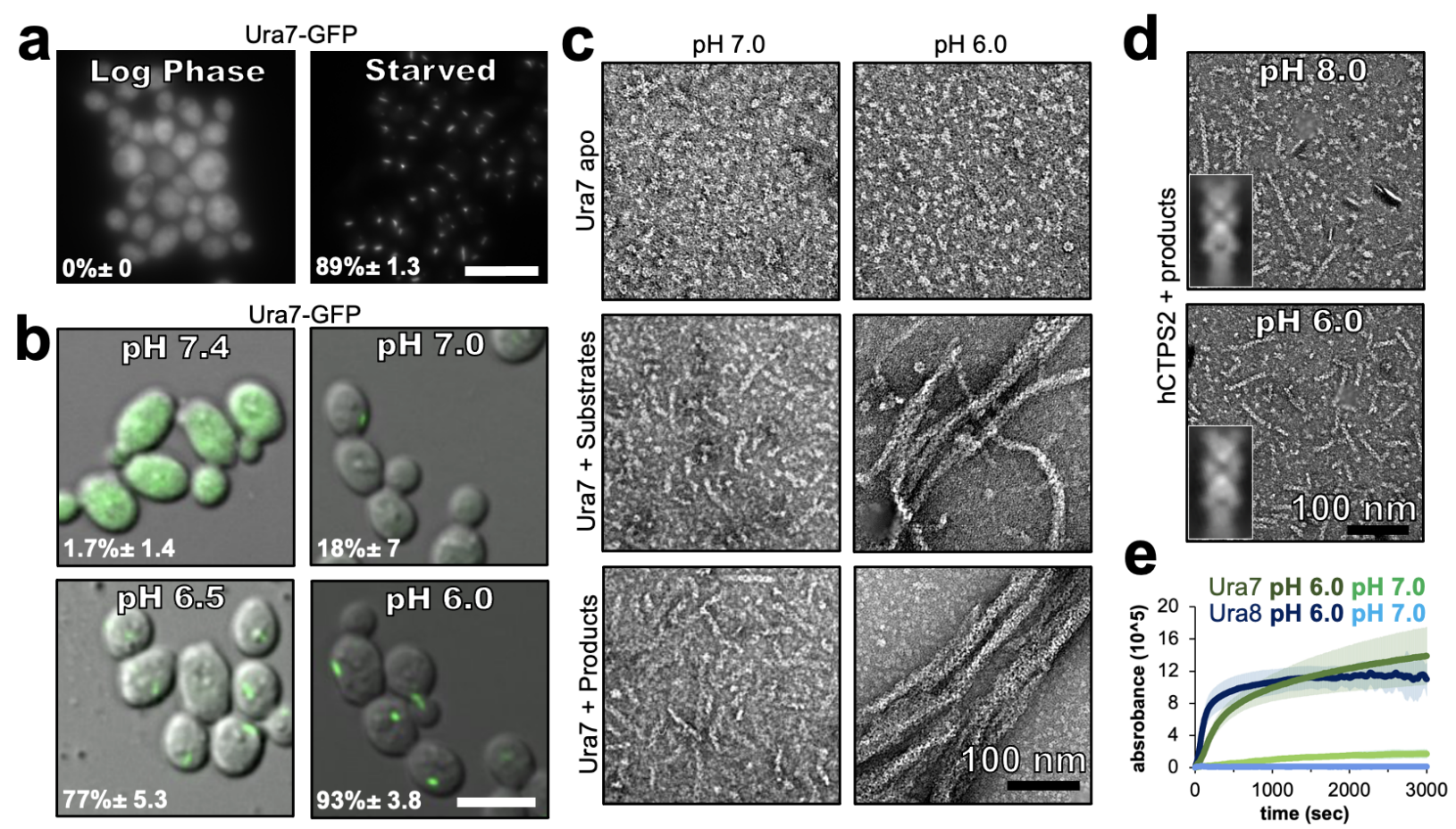

FIGURE 2: Yeast CTPS Assembly is driven by $\mathrm{pH}$ with addition of substrates or products. (a) Yeast expressing GFP-tagged Ura7 in log phase and starvation media. Quantification is shown as a percentage of cells with foci. Scale bar 10 microns (b) Yeast expressing GFP-tagged Ura7 with cell membrane permeabilized using $2 \mathrm{mM}$ DNP supplemented with $2 \%$ glucose. Quantification is shown as a percentage of cells with foci. Scale bar 10 microns. (c) Negative stain EM of purified Ura7 assembled with substrate (2mM UTP/ATP) or products (2mM CTP). (d) Negative stain EM of purified hCTPS2 assembled with 2mM CTP. Insets are representative 2D class averages. (e) Right angle light scattering with addition of $2 \mathrm{mM} \mathrm{CTP.}$

\subsection{Structures of Yeast CTPS filaments}

We next determined structures of individual CTPS filaments by cryo-EM. To enable structure determination, we assembled filaments at $\mathrm{pH} 6.5$, where single filaments predominate over larger bundles. We solved filament structures of both Ura7 and Ura8 in substrate- (ATP and UTP) and product- (CTP) bound states. Ura8 yielded the highest resolution structures, at $2.8 \AA$ (substrates) and $3.8 \AA$ (products) (Fig. 3a, table 1). In the Ura8 structures all ligands were clearly visible and bound as previously described for other CTPS homologs, including an iminophosphate reaction intermediate recently observed in drosophila CTPS, and the presence of two distinct CTP binding sites per monomer recently reported in human and drosophila CTPS (Endrizzi et al., 2004; Goto et al., 2004; Lynch et al., 2017; Lynch and Kollman, 2020; Zhou et al., 2021, 2021, Lynch et al., in review) (Fig3 Supp 1c). Ura7 filaments reached lower resolutions, $7.3 \AA$ (substrates) and $3.7 \AA$ (products), but the overall structures were indistinguishable from Ura8 at these resolutions (Fig3 Supp 2, Table 2). Because of their higher resolution which enabled building of atomic models, we focus subsequent structural interpretation on the Ura8 filaments.

Like other previously reported structures of human, drosophila, and E. coli CTPS, the yeast enzyme assembles as stacked tetramers. However, it does so using a completely different interaction interface, which was the same in both substrate- and product-bound yeast structures (Fig 3b). Helix 356-370 in the glutaminase domain mediates interactions between tetramers, so 
that each protomer is involved in assembly contacts (Fig 3b). His360 interacts with D370 which would be stabilized by protonation of His 360 at low $\mathrm{pH}$, and likely explains the $\mathrm{pH}$-sensitivity of the assembly interaction (Fig 3b, Fig 3 Supp 3). We see density consistent with two rotamer positions of $\mathrm{H} 360$ that may reflect partial protonation of the sidechain at $\mathrm{pH} 6.5$. One rotamer that likely reflects the protonated state interacts with D370, and the other rotamer that likely reflects an unprotonated state forms a hydrogen bond to a backbone carbonyl across the interface (fig 3 Supp3a-c). Additional contacts include hydrogen bonding between an asparagine pair on the two-fold symmetry axis (Asn364), hydrogen bonding (GIn352) to a backbone carbonyl across the interface, hydrophobic interactions of a cluster of tryptophans (Trp363, Trp392), and a pair of salt bridges (Lys391, Glu395) (Fig 3b, Fig 3 Supp 2). Helix 356370 also forms the assembly interface of animal CTPS filaments, but the yeast interface is shifted by two turns of the helix, resulting in completely different residue contacts (Fig $3 \mathrm{c}$ ). This leads to a larger interaction interface in yeast CTPS filaments $\left(706 \AA^{2}\right.$ per monomer in productbound filaments) than in the human homolog ( $492 \AA^{2}$ per monomer in product-bound filaments) (Fig3 Supp 4). Sequence differences between human and yeast CTPS at residues 364, 391, and 395 may explain how the unique yeast interface arose, which shifted H360 into a position to mediate a $\mathrm{pH}$-sensitive interaction.

The primary difference between the substrate- and product-bound filament structures is the conformation of individual CTPS protomers. Active and inactive conformations of CTPS are characterized by $a \sim 7^{\circ}$ rotation of the glutaminase domain relative to the amido-ligase domain, which opens an ammonia channel between the two active sites in a single protomer (Lynch and Kollman, 2020). The Ura8 product-bound filament is in the canonical inhibited conformation, but the substrate-bound structure is in a conformation intermediate between canonical active and inhibited states, in which the ammonia channel remains closed. This suggested to us that one function of yeast CTPS filaments is to constrain the enzyme in a low activity conformation. To test this hypothesis, we determined the structure of free Ura8 tetramers at $\mathrm{pH} 7.4$ bound to substrates at $2.8 \AA$ resolution. In this unassembled state, Ura8 adopts the canonical active conformation, very similar to hCTPS2, including opening of the ammonia channel and rearrangement at the tetramerization interface (Fig. 3d-h, Fig. 3 Supp 5, movie 1).

The active state we observe in free tetramers is incompatible with filament assembly, because in this conformation filament interfaces cannot be occupied on both sides of the tetramer simultaneously (Fig3 Supp 6). Indeed, the major source of heterogeneity in single Ura7substrate filaments, which were flexible and short, are tetramers that only make one contact at each paired assembly interface. This leads to tetramers in the active, substrate-bound conformation tethered to each other through single glutaminase domain interactions (Fig3 Supp $6 \mathrm{~d})$. Thus, filament assembly with symmetric contacts acts as a steric constraint to trap yeast CTPS in a low activity conformation, while tethering through a single glutaminase domain supports short, flexible filaments that accommodate a fully active conformation. 

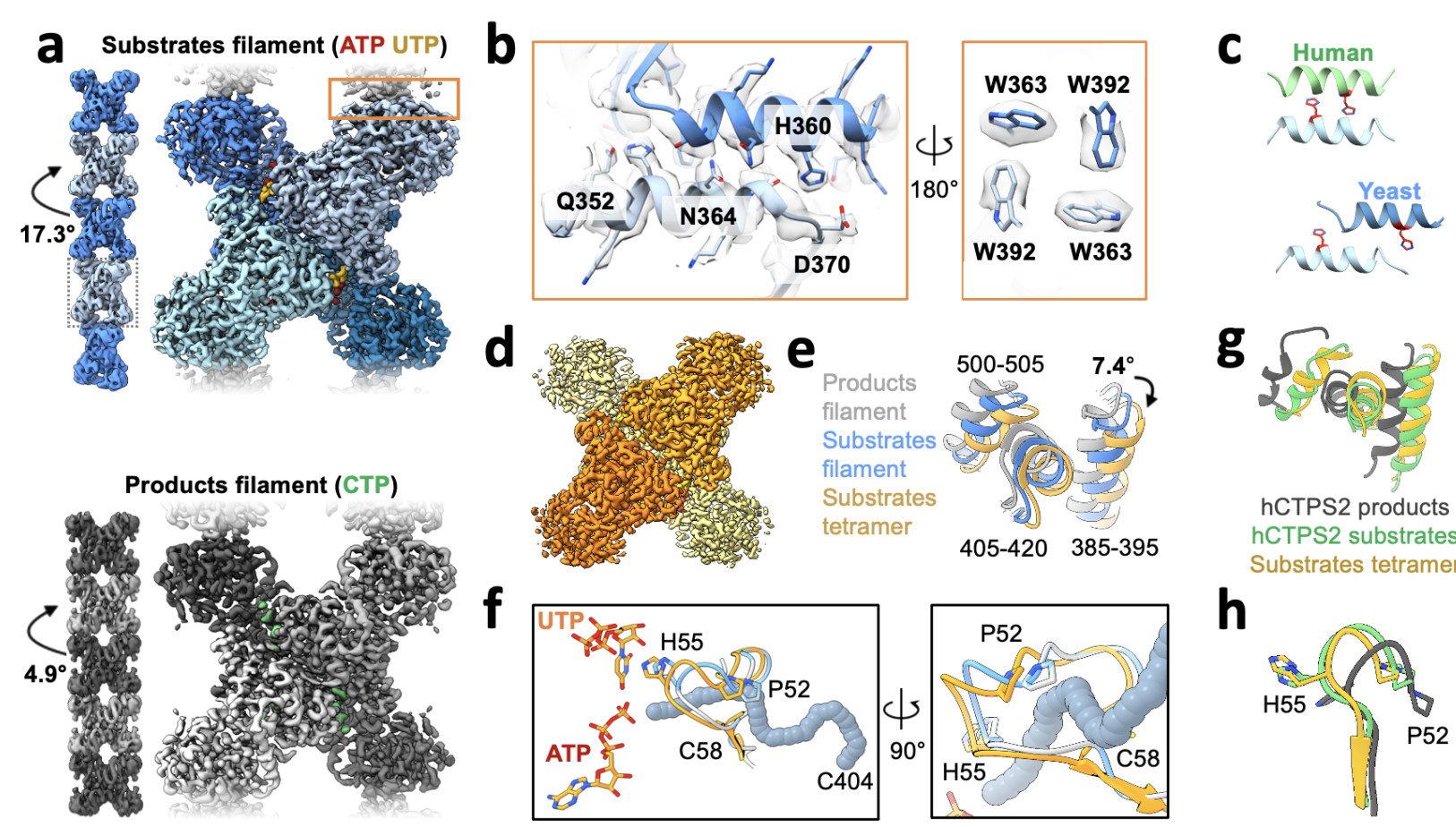

hCTPS2 products hCTPS2 substrates Substrates tetramer

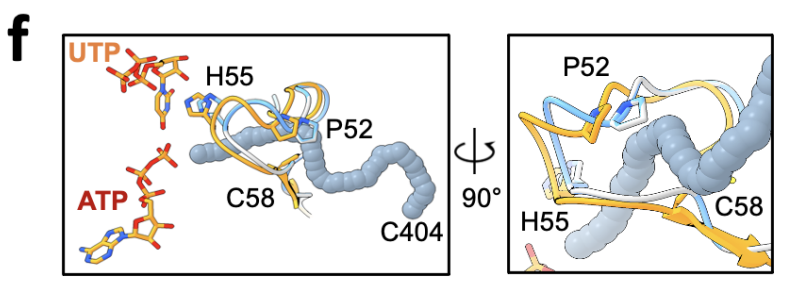

h

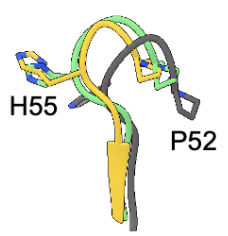

FIGURE 3: Yeast CTPS assembles distinct filaments which are not in the canonical active conformation. (a) Cryo-EM maps of Ura8 filaments assembled at $\mathrm{pH} 6.0$ in the presence of substrates (top; UTP and ATP) or product (bottom; CTP). Left, filament map from imposing helical symmetry parameters on a reconstruction of a single protomer, and low-pass filtered to $15 \AA$. Dotted box delineates an individual tetramer. (b) Zoom-in of the filament assembly interface (orange box in panel A) with superimposed cryo-EM density. Key residues are indicated. Right panel is the back-side view of the interface. (c) Comparison of human and yeast interface, with H360 (H355 in humans) highlighted in red. (d) Cryo-EM map of substrate-bound (ATP,UTP) tetramer at $\mathrm{pH}$ 7.4. (e) Glutaminase domain rotation (residues indicated) of monomers aligned on the amido-ligase domain. (f) Substrate-channel (grey tube) beginning at catalytic C404 ending at ligands UTP/ATP. Color scheme same as panel E. (g) Ura8 substrate-bound tetramer glutaminase domain rotation relative to hCTPS2 substrate (6PK4) and product-bound (6PK7) filaments. (h) Loop movements near the active site which alleviates constriction in the substratechannel. Color scheme same as panel $\mathrm{G}$.

\subsection{Yeast CTPS filaments reduce enzyme activity}

To test whether CTPS filament assembly has a direct effect on enzyme activity, we generated mutations at $\mathrm{H} 360$ in the assembly interface that either disrupted or stabilized filaments. To break assembly we made $\mathrm{H} 360 \mathrm{~A}$, which disrupts polymerization of the human enzymes, and which our structural analysis suggests is the $\mathrm{pH}$ sensor in assembly of the yeast enzyme (Lynch et al., 2017; Lynch and Kollman, 2020). To mimic protonation at this site, we mutated $\mathrm{H} 360$ to arginine with the expectation that a constitutive positive charge might induce polymerization at neutral $\mathrm{pH}$. Recombinant wildtype and mutant proteins had similar purity and yield (Fig 4 Supp1a). Both engineered mutations behaved as designed in terms of filament assembly: $\mathrm{H} 360 \mathrm{~A}$ prevents assembly at all $\mathrm{pH}$ values tested, while H360R robustly assembles filaments at 
high pH (Fig 4a; Fig 4 Supp2). To confirm that H360R does not alter filament structure, we determined a $6.7 \AA$ cryo-EM structure of substrate-bound Ura7, which is indistinguishable from wildtype at this resolution (Fig4 Supp 1b-f).

Decoupling polymerization from $\mathrm{pH}$ in engineered mutations provides tools to determine the functional consequences of polymerization on enzyme activity. Yeast CTPS activity has a strong intrinsic pH-dependence that peaks around pH 8.0 (Nadkarni et al., 1995; Yang et al., 1994), likely due to $\mathrm{pH}$ sensitivity of the active site cysteine in the glutaminase domain (Trotta et al., 1973). Consistent with this, Ura7 wildtype, H360A, and H360R all have low activity at pH 6.0. However, H360A had approximately 3-fold higher activity, indicating that unassembled CTPS maintains residual activity even at low $\mathrm{pH}$ (Fig 4b). Wildtype CTPS and mutants retain activity at $\mathrm{pH} 7.4$, where we measured the substrate kinetics of all three. The $\mathrm{K}_{M}$ values were similar for wildtype and both mutants, indicating no change in affinity for the substrate UTP. However, $V_{\max }$ varied inversely with the degree of filament assembly (Fig. 4c; Table 3). At pH 7.4 Ura7-H360A is completely tetrameric and had the highest activity, wildtype enzyme has a background of single filaments and had slightly lower activity, and Ura7-H360R had robust filament assembly and the lowest observed activity. This suggests that filament assembly in itself acts as an allosteric inhibitor of enzyme activity, with kcat reduced likely as a consequence of the constricted ammonia channel we observe in the filament structure.
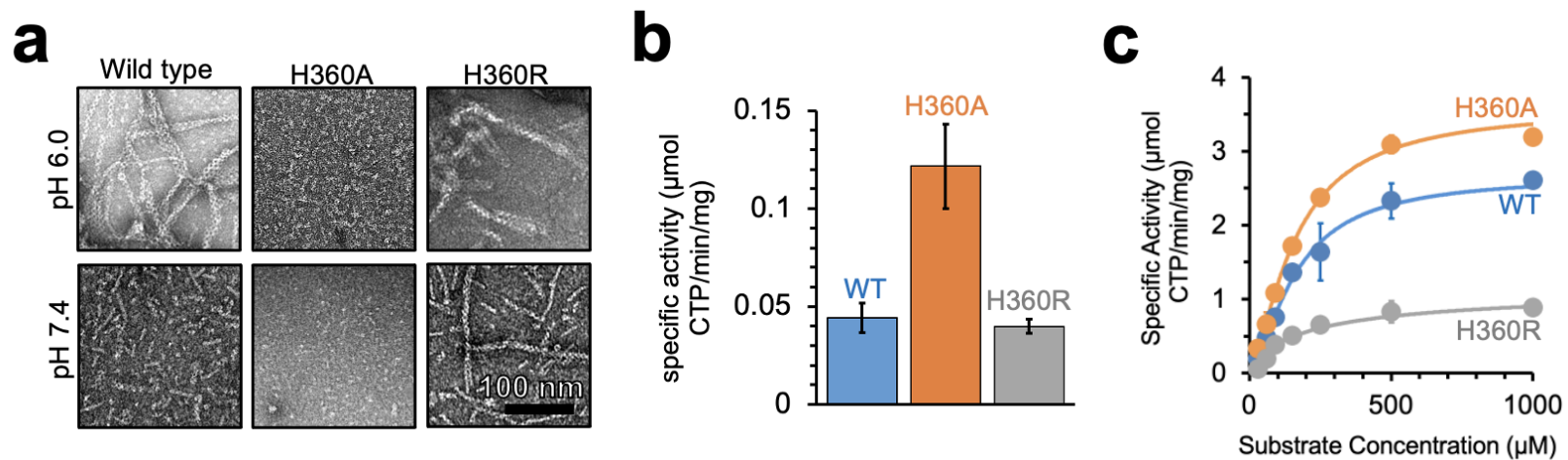

Figure 4. Yeast CTPS polymers reduce enzymatic activity. (a) Negative stain EM of purified wild type and mutant Ura7 with addition of $2 \mathrm{mM} \mathrm{CTP}$. (b) Activity assay with saturating substrates of Ura7 and mutants at $\mathrm{pH} 6.0$. (c) Substrate kinetics of wild type and mutant Ura7 at pH 7.4 with four parameter regression curve fits. Experiments done in triplicate, error bars are standard error of the mean (SEM).

\subsection{CTPS assembly is critical for growth}

To investigate the in vivo consequences of CTPS assembly, we generated yeast strains with filament assembly mutations at the endogenous locus, either with or without fluorescent protein tags, and tested both enzyme localization and cell growth (Fig 5a). We did not observe differences in growth between wildtype strains with and without fluorescent tags; nonetheless, the growth assays described below were all performed with untagged strains. 
Based on our in vitro findings, we predicted that the hyper-polymerizing Ura7-H360R would constitutively assemble polymers in cells. But surprisingly, its localization was very similar to wildtype, diffuse during log phase growth and assembled into foci upon nutritional deprivation. However, Ura7-H360R-GFP assembly and disassembly kinetics are dysregulated (Fig. 5b-c, Fig 5 Supp1, movies 2-5). Upon transition to starvation media, wildtype Ura7-GFP typically assembles foci over the course of 30 minutes, but Ura7-H360R-GFP assembles more rapidly, with virtually all cells having foci at 5 minutes. Even more striking, upon recovery from starvation, in which wildtype Ura7 disassembles in most cells after 30 minutes, at one hour we did not observe significant depolymerization of Ura7-H360R-GFP foci (Fig 5 Supp1). The rapid assembly and slow disassembly of Ura7-H360R in cells are consistent with the in vitro hyperpolymerizing phenotype, but what prevents constitutive assembly during log phase growth is not clear. It suggests that the starvation-triggered $\mathrm{pH}$ change is necessary but not sufficient to enable cellular Ura7 assembly. In addition to disrupted assembly kinetics, Ura7-H360R grew more slowly than wildtype, when plated either from log phase cultures or from starved cultures (Fig. 5d-e), suggesting that disruption of normal Ura7 assembly and disassembly is generally detrimental to growth.

Consistent with our in vitro findings, cells expressing non-assembling Ura7-H360A-GFP or Ura8-H360A-GFP did not form foci, even under nutritional stress when the cytoplasm is acidified (Fig. 5a). The non-assembly single mutants of Ura7 or Ura8 grew indistinguishably from wild type during log phase and upon recovery from starvation (Fig. 5d-e; Fig5 Supp2). This suggested that polymerization of either CTPS isoform is sufficient to maintain normal growth. To test this, we generated the double mutant URA7-H360A/URA8-H360A, and found that it had a severe growth defect in log phase and upon starvation recovery (Fig $5 d, e$ ), indicating an important role for CTPS polymerization in proliferation.

We wondered whether filaments might play a role in protecting CTPS from degradation, and that perhaps the growth defect in the double H360A mutants is due to loss of the enzyme during starvation (Petrovska et al., 2014). To test this, we starved Ura7-GFP, Ura7-H360A-GFP, and Ura7-H360R-GFP strains and measured the concentration of CTPS in total cell lysate. Wildtype Ura7 levels are decreased during starvation, but there was no difference in protein levels between wild type and the mutants at 4 or 24 hours of starvation (Fig. 5 Supp3). This indicates that filament assembly does not play a significant role in protecting CTPS from degradation, and suggests that growth defects observed upon starvation recovery for URA7-H360A/URA8-H360A arise from other effects of defective polymerization.

It was surprising that strains with $\mathrm{H} 360 \mathrm{~A}$ or $\mathrm{H} 360 \mathrm{R}$ mutations experienced slow growth relative to wildtype during log phase, when the mutant strains have the same diffuse localization seen in the wildtype strain. This may be consistent with the observation that wildtype enzymes have a low level of background assembly at neutral pH (Fig. 2c), and indicates that small, transient assemblies may play a role in regulating activity during log phase growth. 

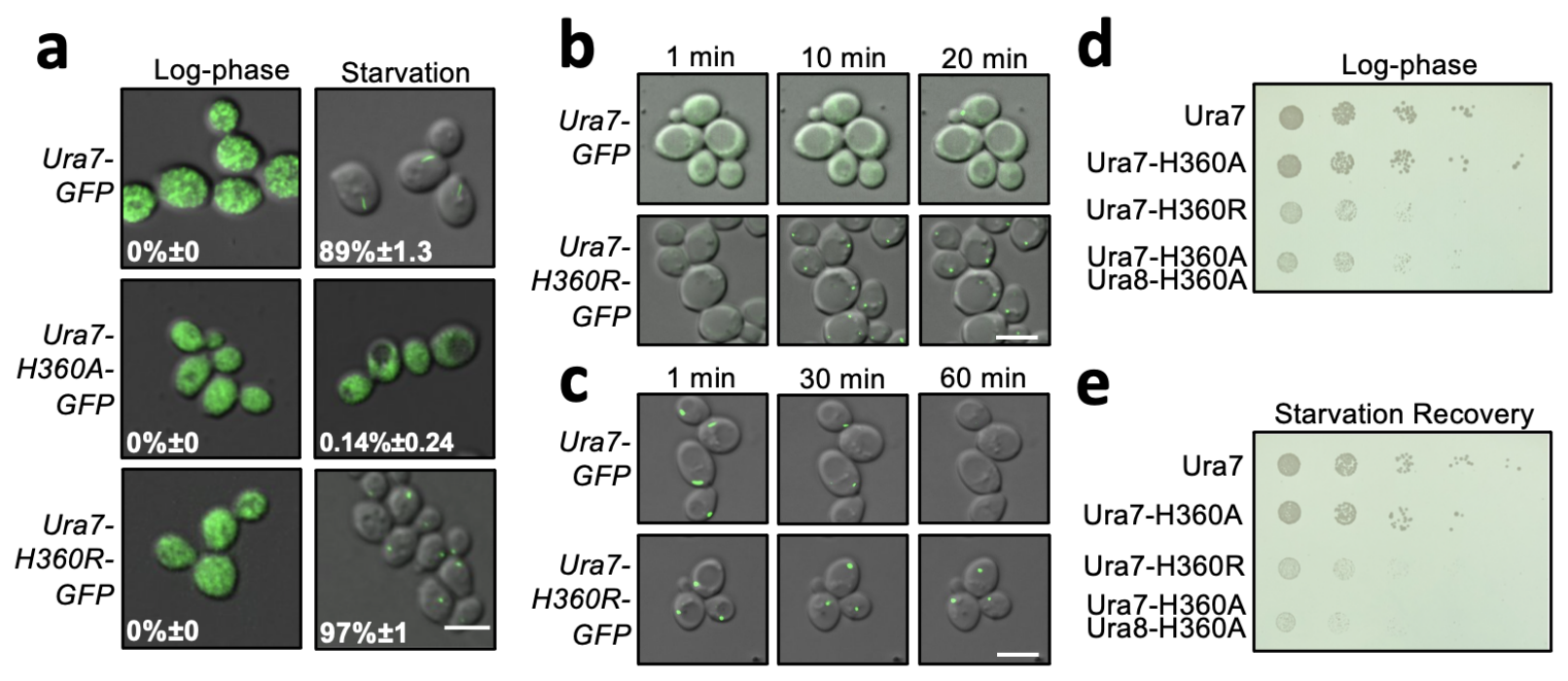

Figure 5. Dysregulated assembly of CTPS in yeast leads to slowed growth. (a) Yeast expressing GFP-tagged Ura7. Quantification is the percentage of cells showing foci. Scale bar is 5 microns. (b) Polymerization kinetics of GFP-tagged Ura7 in yeast upon transfer of cells to starvation media. (c) same as B, for depolymerization kinetics upon re-addition of starved cells (4 hours) to nutrient-rich media. Scale bar is 5 microns. (d) Spot growth assay of yeast expressing wild type and mutant CTPS from liquid cultures grown in log phase. 1 in 10 dilutions increase from left to right. (e) Same as D, for yeast starved for 4 hours and allowed to recover on nutrient rich solid media.

\subsection{Yeast CTPS assembles large-scale ordered bundles}

In wildtype cells at 30 minutes after nutrient deprivation, cells that had CTPS foci almost all had just a single structure (Fig 2b), consistent with prior reports of CTPS polymerization in yeast (Noree et al., 2010; Petrovska et al., 2014; Shen et al., 2016). However, during the live cell imaging experiments described above, in cells expressing wildtype Ura7-GFP we frequently observed the appearance of multiple small puncta at early time points that later coalesced into a single large structure (Fig 6a). This in vivo behavior is reminiscent of the rapid linear polymerization followed by lateral aggregation into larger bundles that we observed in vitro (Fig 2 Suppl 2).

We determined structures of CTPS filament bundles formed at $\mathrm{pH} 6.0$ to better understand the mechanisms of lateral assembly. Our initial question was whether the bundles were aggregating non-specifically, or whether there were defined assembly contacts. Reference-free twodimensional averages of bundle segments from cryo-EM images of Ura7 and Ura8 were strikingly regular, suggesting ordered assembly contacts. This high degree of order allowed us to determine three-dimensional structures of both homologs in the presence of substrates or product using a single particle reconstruction approach by focused refinement of interacting pairs of filaments (Fig 6b and Fig6 Supp1, workflow). As we found for the single filament structures, Ura8 reconstructions went to higher resolution ( $3.3 \AA$ for substrates and $3.7 \AA$ for product) than Ura7 (6.6 $\AA$ substrate and $7.0 \AA$ product). The individual filaments that pack laterally in the bundles closely resemble the corresponding single filament structures (Fig 6 
Supp 2, table 2). We observed two different bundle architectures. Ura7 formed very similar bundles in both ligand conditions, with adjacent filaments staggered relative to each other with a half-tetramer offset (Fig 6c). The Ura8 substrate-bound structure had the same architecture as both Ura7 bundles, but the product-bound Ura8 structure had very different interfaces between filaments, giving rise to filaments in register.(Fig 6d, movies 6-9).

To better understand the nature of lateral assembly contacts, we built atomic models into the two Ura8 bundle structures. Ligands were clearly visible, and bound as in the single filament structures, and the longitudinal assembly interfaces were the same as observed in the single filaments (Fig 6 Supp 2). Domain rotations and the state of the closed ammonia channel were nearly identical between filaments and bundles (Fig 6 Supp 3). Unlike single Ura8 filaments determined at an intermediate $\mathrm{pH}(6.5)$ and with two rotamers for His360, the bundle map appeared to fit a single rotamer which points to D370 (Fig 3 Supp 3d-e). Lateral associations between filaments result in tightly packed bundles. The buried surface area per tetramer at lateral interfaces, $2202 \AA^{2}$ (substrates-bound) or $1676 \AA^{2}$ (product-bound), is comparable to the $2460 \AA^{2}$ involved in longitudinal filament assembly contacts, suggesting that lateral association contributes to the overall stability of the assembly (table 4). Yeast CTPS has a 7-residue insert (residues 273-279) in the linker region that mediates the bulk of the lateral interaction in both bundle types (Fig 6e). In the substrate-bound structure, Leu277 nestles into a hydrophobic pocket formed by lle383/lle387//le412/Phe429 (Fig 6f; Fig6 Supp 4a-b). In the product-bound structure the linker inserts near the tetramerization interface and packs against Pro236 and Ile225 (Fig6 Supp 4c-d). Although at lower resolution, the Ura7 structures appear to make the same lateral contacts as substrate-bound Ura8 (Fig6 Supp 1). Similar filament bundles have not been reported for other species, despite extensive structural characterization of human, drosophila, and E. coli CTPS filaments (Barry et al., 2014; Lynch et al., 2017; Lynch and Kollman, 2020; Zhou et al., 2021), suggesting that the unique yeast insert promotes lateral assemblies that are specific to yeast.

Although individual filament-to-filament lateral interactions are all identical, there is variability of the assembly architecture at longer scales. The dihedral symmetry of the CTPS tetramer presents two potential lateral interfaces on each face of single filaments, but steric constraints limit occupancy to just a single lateral interaction per face. 3D classification of bundles yielded multiple structures with 3-5 associated filaments, with mixtures of cis or trans configurations of laterally associated filaments accounting for the variation (methods flowchart). To envision potential larger bundle architectures we extrapolated the lateral contacts by propagating assemblies in silico (Fig6 Supp 5). For the staggered bundle architecture, propagation of cis or trans interactions results in curved sheets. Propagation of trans interactions in the Ura8 product bound structure with filaments in register also results in a curved sheet, but propagation of cis interactions results in a closed tube with nine filaments. Mixed cis and trans interactions are also possible, and observed in some of our 3-D classes, and give rise to increasingly complex structures. 


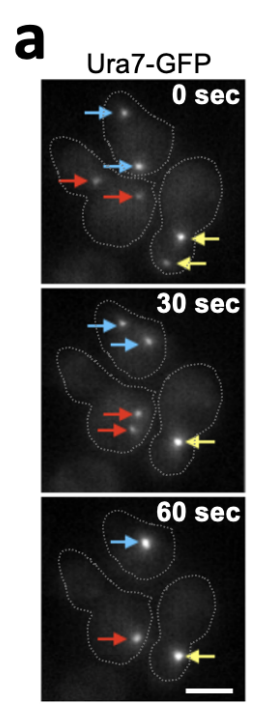

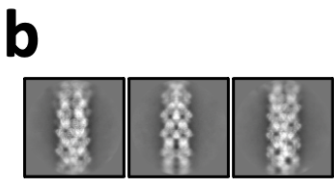

Ura8 Substrates

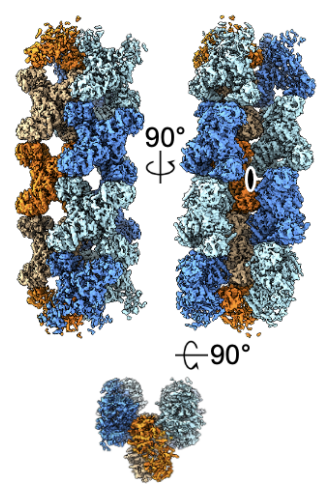

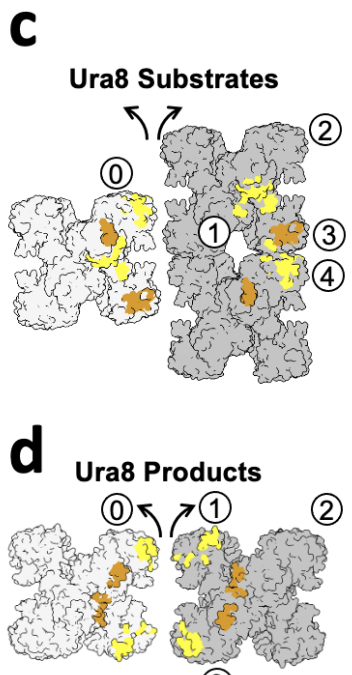

(3)

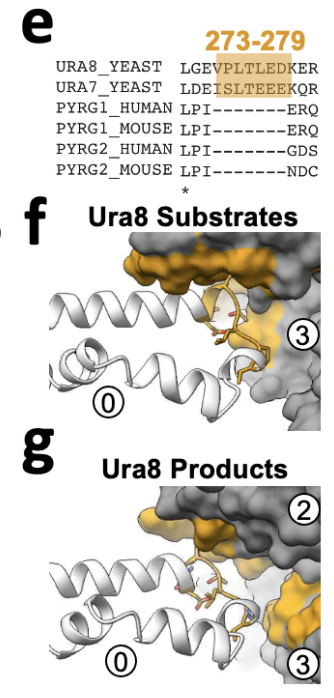

FIGURE 6: Yeast CTPS Assembles Highly Ordered and Distinct Bundle Architectures (a) Live imaging in yeast expressing GFP-tagged Ura7. Same colored arrows indicate pairs of foci that join to larger foci at later time points. Time indicated is seconds after imaging began. Cell outlines drawn manually. Scale bar 5 microns. (b) Cryo-EM 2D averages and 3D reconstructions of Ura8 in the substrate (2mM UTP/ATP) and product-bound (2mM CTP) states. Individual strands colored in either orange or blue with protomers shaded differently. C2 symmetry axes are shown as ovals, with axis of rotation projecting towards the reader. (c) Lateral interactions in the substrate-bound Ura8 bundle. Full contacts are painted yellow for a single tetramer (grey on left), including redundant interacts. The yeast-specific linker insert and the interface with which it interacts are painted brown. Unique contacts for a single monomer " 0 " interacts with four monomers "1-4" on the neighbor strand (see table 4). (d) Same as C, for product-bound Ura8 bundle. Unique interactions are for a single monomer "0" interacting with three monomers "1-3" on the neighbor strand (see table 4). (e) Sequence alignment of the CTPS linker region showing the yeast-specific insert at the specified yeast residues. (f) Zoom-in of the yeast linker insert of the substrate-bound bundles interacting with the adjacent strand. Numbering is the same as panel C. (g) Same as panel F, for product-bound bundle. Numbering is the same as panel D.

\section{Discussion}

Maintenance of balanced nucleotide pools is essential for all organisms, and CTPS plays a critical, conserved role in directly balancing pyrimidine levels. The polymerization of CTPS into cellular filamentous polymers occurs in bacteria, archaea, and in eukaryotes (Carcamo et al., 2011; Ingerson-Mahar et al., 2010; Liu, 2010; Noree et al., 2010; Zhou et al., 2021). Bacterial and animal CTPS filaments assemble with completely different interfaces and functional consequences for enzyme regulation, inhibiting activity in the bacterial enzymes and increasing activity or enhancing cooperative regulation in the animal enzymes (Barry et al., 2014; Lynch et al., 2017; Lynch and Kollman, 2020). We have shown that budding yeast CTPS assembles filaments with yet another, distinct assembly interface that maintains the enzyme in a low activity state. The diversity of CTPS filament structure and function raises the question of whether this represents modification of an ancestral filament structure, or whether CTPS polymerization has evolved independently multiple times. The latter would be consistent with the 
observation that proteins with dihedral symmetry (like the CTPS tetramer) can be induced to polymerize with a very small number of mutations on their high symmetry surfaces (GarciaSeisdedos et al., 2017). Given the regulatory importance of CTPS in nucleotide homeostasis, polymerization may have evolved as a relatively straightforward way to introduce a new layer of allosteric regulation to meet different demands in different lineages.

Whatever its evolutionary origins, the yeast CTPS filament has acquired features that make its assembly responsive to cytoplasmic changes in nutrient availability and growth conditions. Upon starvation-induced cytoplasmic acidification, CTPS assembly is dramatically increased in cells (Noree et al., 2019; Petrovska et al., 2014; Shen et al., 2016). We have shown here that yeast CTPS polymerization is a self-assembly mechanism that does not require other cellular factors, and that the $\mathrm{pH}$-sensitivity of CTPS polymers is intrinsic to the enzyme itself (Fig 2c). The unique yeast CTPS assembly interface positions a titratable histidine residue, $\mathrm{H} 360$, to interact with an acidic residue, D370, an interaction that is likely strengthened at lower $\mathrm{pH}$ by $\mathrm{H} 360$ protonation (Fig 3 Supp 3). In the filament, CTPS is held in a conformation that closes off an internal ammonia channel that likely reduces activity (Fig 3f,h). Mutations at the assembly interface that either block assembly (H360A) or eliminate the $\mathrm{pH}$ sensitivity (H360R) showed that assembly is correlated with reduced activity, both at neutral $\mathrm{pH}$ where the wildtype enzyme forms short single polymers, and at low $\mathrm{pH}$ where the wildtype enzyme forms large laterally assembled bundles that are three-fold less active than the non-assembling mutant (Fig 4b).

Highly ordered, laterally-associated filament bundles are another unique feature of yeast CTPS not observed in CTPS filaments of other organisms. A yeast-specific insert in the linker domain mediates the lateral contact, suggesting an organism-specific need to organize CTPS assemblies in yeast. Ura7 bundles in either ligand state are very similar, and at our resolution appear to maintain the same lateral contacts but with a slightly different helical twist.

Regardless of nucleotide pools, bundles could therefore remain assembled to fulfill their role in the starvation response. Conversely, Ura8 forms bundles with radically different architectures dependent on whether substrates or products are bound; it remains unclear what the functional consequence is of having two distinct bundle architectures. Both types of bundles are able to accommodate lateral addition of strands in various ways, and this heterogeneity suggests that the specific architecture may be of less importance than simply maintaining protomers in a polymer.

The CTPS reaction mechanism is intrinsically sensitive to $\mathrm{pH}$, with reduced activity at lower $\mathrm{pH}$ (Nadkarni et al., 1995; Yang et al., 1994). What then is the utility of having a redundant allosteric mechanism for inhibition by filament assembly at low $\mathrm{pH}$ ? One possibility may be that the low residual activity of tetrameric CTPS at low $\mathrm{pH}$ is sufficient to imbalance nucleotide pools over prolonged starvation. Another explanation may lie in the kinetics of reactivation. Upon reintroduction of glucose the cytoplasm returns to neutral $\mathrm{pH}$ over about 2 minutes (Orij et al., 2009), but CTPS filaments disassemble over about 30 minutes or longer (Fig 5c; Fig 5 Supp1), which may enable a more controlled ramping up of enzyme activity upon re-entering growth. In both our non-assembling and hyper-assembling mutants this process of controlled disassembly is disrupted, resulting in severe growth defects.

Our in vitro results correlating $\mathrm{pH}$, filament assembly, and activity levels suggest a model for cellular function for CTPS filaments. Under growth conditions near neutral pH CTPS filaments 
modulate activity through an equilibrium of unassembled tetramers and dynamic short filaments that would be responsive to transient changes in substrate and product pools, as has been proposed for CTPS filaments of other species (Barry et al., 2014; Lynch et al., 2017). Sensitivity of CTPS polymers to the balance of substrates and products modulates transient spikes in UTP or CTP concentrations by driving assembly of inactive filaments or disassembly into active tetramers and loosely tethered flexible filaments (Fig 7a). Upon starvation, however, acidification acts as a signal to strongly drive assembly and inactivate the entire pool of CTPS in preparation for entering quiescence. Continued acidification drives filament growth as enzymatic activity ramps down, and eventually association of filaments into bundles (Fig $7 \mathrm{~b}$, center) and lateral interactions maintain filaments in an assembled, low activity state. Upon nutrient readdition and rapid re-alkalinization of cytoplasm, the size of bundles leads to a lag in depolymerization and a gradual ramp up of activity towards log-phase growth (Fig 7b, right).

Disruption of polymerization-based allosteric regulation of CTPS likely results in disruption of nucleotide homeostasis, with potential consequences for processes that depend on nucleotide pools like ribosome biosynthesis, DNA replication, and phospholipid biosynthesis (Chang and Carman, 2008; Fairbanks et al., 1995). We predict that non-assembling mutants would overproduce CTP and deplete the substrate UTP, while hyper-assembling mutants would decrease CTP production, with cascading consequences for connected metabolic pathways. Further, disruption of CTPS increases genomic instability (Whelan 1993), and specifically disrupting Ura7 in yeast is strongly mutagenic, particularly during the stress response (Schmidt et al., 2017). Our characterization of these mutants lays the groundwork for future studies to examine the role of CTPS assembly in maintaining global metabolite levels and flux in pyrimidine biosynthesis.

The assembly defective mutants had localization patterns in cells that were consistent with their in vitro phenotype (Fig 5a). Ura7-H360A-GFP did not form large scale cellular assemblies under any growth conditions, confirming that the filament interface we observe in cryo-EM structures is important for assembly in vivo. Ura7-H360R-GFP, on the other hand, did not assemble large structures during log phase growth, as we would have predicted based on our observation of in vitro assembly at neutral $\mathrm{pH}$ (Fig 5a). However, there was a striking difference in in vivo assembly kinetics of Ura7-H360R-GFP which, unlike the wildtype enzyme, assembled much more rapidly than the approximately 30-60 minutes it takes for cytoplasmic acidification upon nutrient deprivation (Orij et al., 2009) (Fig 5b, Fig 6 Supp 1). This suggests to us that in the cellular context some factor in addition to $\mathrm{pH}$ is preventing assembly during log phase growth, and once this block is lifted upon nutrient deprivation the Ura7-H360R-GFP polymer rapidly assembles even before the cytoplasm has acidified. Such a licensing mechanism might prevent small oscillations in nutrient availability from triggering massive CTPS assembly by transient $\mathrm{pH}$ changes, but only allow assembly under more pronounced stress. One candidate for such a licensing event could be phosphorylation or dephosphorylation of CTPS. Several functionally important phosphorylation sites have been identified in yeast CTPS (Choi et al., 2003; Park et al., 2003, 1999), one of which is near a bundle assembly contact (S354 in Ura7).

Our findings do not rule out other functions for CTPS filaments beyond a role in allosteric enzyme regulation. In particular, one possibility is that filament formation by multiple yeast enzymes during starvation changes physical properties of the cytoplasm to a more protective solid-like state (Petrovska et al., 2014). CTPS filaments likely do contribute to bulk cytoplasmic 
changes, but our observation that the disruption of only this filament is sufficient to drastically disrupt normal growth suggests that CTPS polymers play a more specific role in managing nutrient stress. CTPS filaments may also serve other functions in scaffolding or signalling; Ura7 co-localizes with other metabolic filaments in yeast (Noree et al., 2019, 2010), raising the possibility that filaments provide a mechanism for direct physical interaction of enzymes for coordinated regulation of different pathways. Alternatively, bundles may serve as a signalling mechanism of nutrient deprivation for interacting partner proteins. Previous studies have identified eif2 translation initiation factor interaction with CTPS filaments in drosophila, which may have direct consequences on growth through organizing protein expression (Zhang et al., 2021). Future studies looking at the co-assembly state of these enzymes in the context of CTPS assembly mutants will be informative for determining whether CTPS bundles play an additional role in the stress response. 


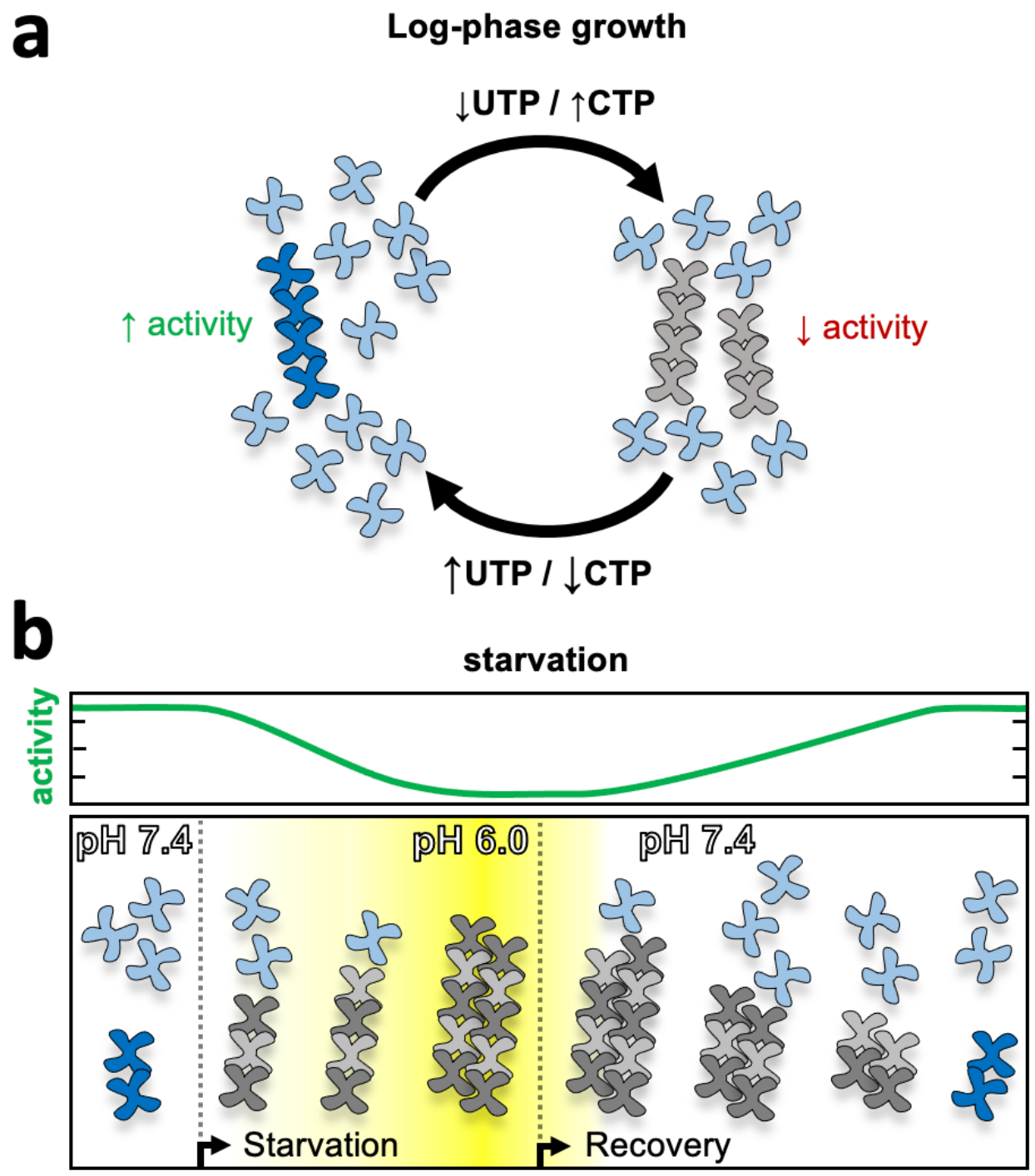

Figure 7. Model of yeast CTPS filament Function. (a) log-phase growth in yeast the majority of CTPS is in the unassembled active conformation, with a subset of enzyme polymerized to maintain nucleotide pool balance. If UTP levels are high, filaments are in the strained substratebound conformation which breaks assembly contacts, allowing the enzyme to remain active and process substrate while polymerized. When CTP levels are high, filaments assemble rigid product-bound filaments which inactivates the enzyme until equilibrium is reached and CTPS return to the substrate-bound filament state. (b) During log growth there is an equilibrium of CTPS tetramers and filaments (left). Starvation induces a slow cytoplasmic acidification which, in conjunction with a licensing event, leads to CTPS filament and bundle growth. Bundles maintain a low activity state by stabilizing filaments through lateral assembly. As yeast recover from starvation the cytoplasm is rapidly alkalinized, but bundles persist as they slowly disassemble and gradually ramp up activity towards log phase growth. 


\section{Materials and Methods}

\section{Key Resources Table}

\begin{tabular}{|c|c|c|c|c|}
\hline $\begin{array}{c}\text { Reagent, } \\
\text { Species, or } \\
\text { Resource Type }\end{array}$ & Designation & Source/Reference & Identifier & $\begin{array}{l}\text { Additional } \\
\text { Information }\end{array}$ \\
\hline $\begin{array}{l}\text { chemical compound } \\
\text { or drug }\end{array}$ & LB Broth & Lab Express & 3003 & \\
\hline $\begin{array}{l}\text { chemical compound } \\
\text { or drug }\end{array}$ & YPD Media & Lab Express & 3011 & \\
\hline $\begin{array}{l}\text { chemical compound } \\
\text { or drug }\end{array}$ & IPTG & GoldBio & I2481C100 & \\
\hline $\begin{array}{l}\text { chemical compound } \\
\text { or drug }\end{array}$ & $\begin{array}{c}\text { Dextrose } \\
\text { Monohydrate }\end{array}$ & Fisher Scientific & $77938-63-7$ & \\
\hline $\begin{array}{l}\text { chemical compound } \\
\text { or drug }\end{array}$ & $\mathrm{MgCl} 2$ & Fisher Scientific & BP215-500 & \\
\hline $\begin{array}{l}\text { chemical compound } \\
\text { or drug }\end{array}$ & $\mathrm{NaCl}$ & Fisher Scientific & S271-10 & \\
\hline $\begin{array}{l}\text { chemical compound } \\
\text { or drug }\end{array}$ & $\mathrm{KCl}$ & Fisher Scientific & BP217-3 & \\
\hline $\begin{array}{l}\text { chemical compound } \\
\text { or drug }\end{array}$ & Imidazole & Sigma Aldrich & SLBT7469 & \\
\hline $\begin{array}{l}\text { chemical compound } \\
\text { or drug }\end{array}$ & HEPES & Fisher Scientific & BP310-1 & \\
\hline $\begin{array}{l}\text { chemical compound } \\
\text { or drug }\end{array}$ & MES & Sigma Aldrich & M8250-100G & \\
\hline $\begin{array}{l}\text { chemical compound } \\
\text { or drug }\end{array}$ & Tris Base & Fisher Scientific & BP152-5 & \\
\hline $\begin{array}{l}\text { chemical compound } \\
\text { or drug }\end{array}$ & ATP & Sigma Aldrich & A2383-10G & \\
\hline $\begin{array}{l}\text { chemical compound } \\
\text { or drug }\end{array}$ & UTP & Sigma Aldrich & U6750-250mg & \\
\hline $\begin{array}{l}\text { chemical compound } \\
\text { or drug }\end{array}$ & CTP & Sigma Aldrich & C1506-250MG & \\
\hline $\begin{array}{l}\text { chemical compound } \\
\text { or drug }\end{array}$ & GTP & Sigma Aldrich & G8877-1G & \\
\hline $\begin{array}{l}\text { chemical compound } \\
\text { or drug }\end{array}$ & Uranyl Formate & $\begin{array}{l}\text { Electron Microscopy } \\
\text { Sciences }\end{array}$ & 22450 & $\begin{array}{c}\text { Negative stain } \\
\text { EM }\end{array}$ \\
\hline $\begin{array}{l}\text { chemical compound } \\
\text { or drug }\end{array}$ & $\begin{array}{l}\text { Kanamycin } \\
\text { Sulfate }\end{array}$ & Acros Organics & 450810500 & \\
\hline $\begin{array}{l}\text { chemical compound } \\
\text { or drug }\end{array}$ & PMSF & Sigma Aldrich & $329-98-6$ & \\
\hline
\end{tabular}




\begin{tabular}{|c|c|c|c|c|}
\hline $\begin{array}{l}\text { chemical compound } \\
\text { or drug }\end{array}$ & Glycerol & Fisher Scientific & G33-1 & \\
\hline $\begin{array}{l}\text { chemical compound } \\
\text { or drug }\end{array}$ & glutamine & Fisher Scientific & BP379-100 & \\
\hline $\begin{array}{l}\text { chemical compound } \\
\text { or drug }\end{array}$ & DTT & Fisher Scientific & $172-25$ & \\
\hline $\begin{array}{l}\text { chemical compound } \\
\text { or drug }\end{array}$ & 2,4-Dinitrophenol & Sigma & $51-28-5$ & $\begin{array}{c}\text { Membrane } \\
\text { permeabilization }\end{array}$ \\
\hline $\begin{array}{l}\text { chemical compound } \\
\text { or drug }\end{array}$ & Agar & Fisher Scientific & BP1423-500 & \\
\hline $\begin{array}{l}\text { Plasmid, } \\
\text { Recombinant } \\
\text { Vector }\end{array}$ & pet28b-6His & Addgene & 73018 & \\
\hline Bacterial Strain & E. coli TOP10 & Thermo Scientific & C404003 & \\
\hline Bacterial Strain & $\begin{array}{l}\text { E. coli BL21 (DE3) } \\
\text { RIL }\end{array}$ & Thermo Scientific & EC0114 & \\
\hline Yeast Strain & W303 & in house & $\begin{array}{c}\text { leu2-3,112 } \\
\text { trp1-1 can1- } \\
100 \text { ura3-1 } \\
\text { ade2-1 his3- } \\
11,15\end{array}$ & \\
\hline EM Equipment & $\begin{array}{l}\text { C-flat } 2 / 2 \text { holey } \\
\text { carbon film }\end{array}$ & Protochips & CF-2/2-2C & \\
\hline other & $\begin{array}{c}\text { superdex } 200 \\
\text { increase } 10 / 300 \mathrm{gl} \\
\text { hi load }\end{array}$ & GE & 28-9893-35 & Gel filtration \\
\hline other & $\begin{array}{l}\text { Amicon Ulta-15 } \\
\text { 30K MWCO } \\
\text { centrifugal filters }\end{array}$ & Millipore & UFC903008 & $\begin{array}{l}\text { Protein } \\
\text { concentrator }\end{array}$ \\
\hline other & $\begin{array}{c}\text { 5-ml HisTrap FF } \\
\text { Crude column } \\
\text { (GE) }\end{array}$ & GE & 17528601 & $\begin{array}{l}\text { Affinity tag } \\
\text { purification }\end{array}$ \\
\hline $\begin{array}{l}\text { Software or } \\
\text { Algorithm }\end{array}$ & MotionCor2 & $\frac{\text { https://doi.org/10.1038/n }}{\underline{\text { meth.4193 }}}$ & & \\
\hline $\begin{array}{l}\text { Software or } \\
\text { Algorithm }\end{array}$ & PHENIX & $\frac{\text { https://doi.org/10.1107/9 }}{\underline{7809553602060000865}}$ & & \\
\hline $\begin{array}{l}\text { Software or } \\
\text { Algorithm }\end{array}$ & CTFFIND4 & $\frac{\text { https://doi.org/10.1016/j.j }}{\underline{\text { sb.2015.08.008 }}}$ & & \\
\hline $\begin{array}{l}\text { Software or } \\
\text { Algorithm }\end{array}$ & gCTF & $\frac{\text { https://doi.org/10.1016/j.j }}{\underline{\text { sb.2015.11.003 }}}$ & & \\
\hline $\begin{array}{l}\text { Software or } \\
\text { Algorithm }\end{array}$ & crYOLO & $\frac{\text { https://doi.org/10.1038/s4 }}{\underline{2003-019-0437-z}}$ & & \\
\hline $\begin{array}{l}\text { Software or } \\
\text { Algorithm }\end{array}$ & Relion & $\frac{\text { https://doi.org/10.7554/e }}{\text { Life.42166 }}$ & & \\
\hline
\end{tabular}




\begin{tabular}{|c|c|c|}
\hline $\begin{array}{l}\text { Software or } \\
\text { Algorithm }\end{array}$ & cisTEM & $\frac{\text { https://doi.org/10.7554/e }}{\text { Life.35383 }}$ \\
\hline $\begin{array}{l}\text { Software or } \\
\text { Algorithm }\end{array}$ & cryosparc & $\frac{\text { https://doi.org/10.1038/n }}{\text { meth.4169 }}$ \\
\hline $\begin{array}{l}\text { Software or } \\
\text { Algorithm }\end{array}$ & Isolde & $\frac{\text { https://doi.org/10.1107/S }}{2059798318002425}$ \\
\hline $\begin{array}{l}\text { Software or } \\
\text { Algorithm }\end{array}$ & Coot & $\frac{\text { https://doi.org/10.1107/S }}{\underline{0907444910007493}}$ \\
\hline $\begin{array}{l}\text { Software or } \\
\text { Algorithm }\end{array}$ & RosettaES & $\frac{\text { https://doi.org/10.1038/n }}{\text { meth.4340 }}$ \\
\hline $\begin{array}{l}\text { Software or } \\
\text { Algorithm }\end{array}$ & UCSF Chimera & $\frac{\text { https://doi.org/10.1002/jc }}{\underline{\text { c. } 20084}}$ \\
\hline $\begin{array}{l}\text { Software or } \\
\text { Algorithm }\end{array}$ & Molprobity & $\frac{\text { https://doi.org/10.1107/S }}{\underline{0907444909042073}}$ \\
\hline
\end{tabular}

Purification of Recombinant CTPS and Mutagenesis. URA7 and URA8 wild type genes with ribosomal binding site were cloned into pet28b-6His (Addgene, Massachusetts; Kan resistance, $\mathrm{C}$-terminal $6 \mathrm{X}$ his tags) at Xhol and $\mathrm{Xbal}$ sites. Mutants were generated by designing semioverlapping primers following quickchange guidelines which incorporated the mutation (Liu and Naismith, 2008). Plasmids were transformed into E. coli BL21 (DE3) RIL cells for expression. Cultures were grown in Luria broth $(\mathrm{LB})$ at $37^{\circ} \mathrm{C}$ until reaching $\mathrm{OD}_{600}$ of 0.8 , then temperature reduced to $18^{\circ} \mathrm{C}$ for induction with $1 \mathrm{mM}$ IPTG overnight. The next morning, cultures were pelleted and either stored at $-80^{\circ} \mathrm{C}$ or protein purification carried out immediately. All subsequent steps were carried out on ice or in a $4^{\circ} \mathrm{C}$ cold room. Pellets from $2 \mathrm{~L}$ of culture were resuspended in $40 \mathrm{~mL}$ ice cold lysis buffer (50mM HEPES, $1 \mathrm{M} \mathrm{NaCl}, 20 \mathrm{mM}$ Imidizole, $10 \%$ glycerol, 1mM PMSF, pH 7.8) and lysed with an Emulsiflex-05 homogenizer (Avestin, Ottawa, Canada) for approximately 5 minutes at $15,000 \mathrm{PSI}$. Lysate was then cleared by centrifugation at $33,764 \mathrm{~g}$ for $30 \mathrm{~min}$ at $4^{\circ} \mathrm{C}$ in a Thermo Scientific Fiberlite F14-14 × 50cy rotor. Clarified lysate was loaded onto a 5-ml HisTrap FF Crude column (GE, Massachusetts) on an ÄKTA start chromatography system (GE) pre-equilibrated with lysis buffer. Unbound material was washed away with 15 column volumes of lysis buffer before an isocratic elution with 5 column volumes of elution buffer (50mM HEPES, $1 \mathrm{M} \mathrm{NaCl}, 250 \mathrm{mM}$ Imidizole, $30 \%$ glycerol, pH 7.8). Peak fractions were combined and concentrated approximately 3-fold using a 30-kDa cut-off Amicon centrifugal filter unit (Millipore, Massachusetts). Approximately $5 \mathrm{~mL}$ of concentrated protein was subjected to size-exclusion chromatography using the Äkta Pure system and a superdex 200 increase 10/300 gl pre-equilibrated with running buffer (50mM HEPES, $1 \mathrm{M} \mathrm{NaCl}, 10 \%$ glycerol, $\mathrm{pH}$ 7.8). Peak fractions were collected and glycerol added to a final concentration of $30 \%$ before again concentrating using a $30-\mathrm{kDa}$ cut-off Amicon centrifugal filter unit. Protein at $\sim 2 \mathrm{mg} / \mathrm{ml}$ was flash frozen in liquid nitrogen and stored at $-80^{\circ} \mathrm{C}$. Recombinant human CTPS2 was purified as described in Lynch 2020.

CTPS activity assays. To reduce variation between wild type and mutants (Ura7 WT, H360A and $\mathrm{H} 360 \mathrm{R}$ ), all three were purified in tandem and flash frozen. Aliquots were thawed, desalted into glycerol-free buffer ( $50 \mathrm{mM}$ Tris $\mathrm{pH} 7.4,200 \mathrm{mM} \mathrm{NaCl}, 10 \mathrm{mM} \mathrm{MgCl}_{2}$ ), and concentrations measured at $A_{280}$. Reactions were set up in 96 well clear plastic flat bottom corning plates 
(Corning, New York) with a $100 \mu$ final reaction volume. For low $\mathrm{pH}$ kinetics, $2 \mathrm{uM}$ protein was incubated with activity buffer (50mM MES pH 6.0, 200mM NaCl, $10 \mathrm{mM} \mathrm{MgCl2,10mM} \mathrm{BME)}$ and nucleotide ( $2 \mathrm{mM}$ ATP, $2 \mathrm{mM}$ UTP, $0.2 \mathrm{mM}$ GTP) for 15 minutes at $30^{\circ} \mathrm{C}$. Reaction was initiated by addition of $10 \mathrm{mM}$ glutamine and absorbance measured at $291 \mathrm{~nm}$ in a plate reader (varioskan lux) set at $30^{\circ} \mathrm{C}$. Early activity traces were noisy, likely due to bundled CTPS, so slope was measured between $350-500$ seconds where rate of activity stabilized. A linear regression was fit to determine slope, which was used to determine CTP concentration. Replicates were performed in triplicate and averaged. Substrate kinetics were run under less optimal conditions in order to capture rates at lowest substrate concentrations. Ura7 at a final concentration of $1.5 \mu \mathrm{M}$ was added to reaction buffer $(50 \mathrm{mM}$ Tris $7.4,200 \mathrm{mM} \mathrm{NaCl}, 10 \mathrm{mM}$ $\mathrm{MgCl}$ ) and nucleotides (1mM ATP, $20 \mu \mathrm{M}$ GTP, with a range of $20 \mu \mathrm{M}$ to $1000 \mu \mathrm{M}$ UTP). Mixture was incubated at room temperature for 5 minutes then inserted into a plate reader set to $22^{\circ} \mathrm{C}$ and allowed to equilibrate for an additional 5-8 minutes, while taking readings at $291 \mathrm{~nm}$ until readouts were stable. The plate was briefly ejected from the reader to manually initiate the reaction by addition of glutamine ( $1 \mathrm{mM}$ final concentration, prepared in $20 \mathrm{mM}$ Tris $\mathrm{pH} 7.0$ ). CTP production was measured at $291 \mathrm{~nm}$ for 5 minutes, capturing the early linear phase of the curve. Assays were performed in triplicate then averaged, and kinetics data were fit by 4 parameter logistic regression, solving for maximum rate, minimum rate, hill number using the solver plugin in Microsoft Excel 16.51.

Right Angle Light Scattering. Frozen CTPS was thawed and desalted into a glycerol-free buffer (200mM NaCl, $20 \mathrm{mM}$ HEPES 7.8). Sample was set up in a total volume of $120 \mu \mathrm{l}$ for assembly at a final protein concentration of $2 \mu \mathrm{M}$ with $1 \mathrm{mM}$ CTP. Assembly buffer was $50 \mathrm{mM}$ MES 6.0 or $50 \mathrm{mM}$ Tris $7.0-7.4,500 \mathrm{mM} \mathrm{NaCl}$, and $10 \mathrm{mM} \mathrm{MgCl}$. Protein was added to the buffer in a quartz cuvette and inserted into a Horiba Fluorolog 3 fluorometer (Horiba, Kyoto, Japan) set to $30^{\circ} \mathrm{C}$ and allowed to incubate until signal stabilized (approximately 3-5 minutes). Assembly was initiated by addition of nucleotide then readings began immediately, usually with a 5 second lag between addition and commencing readings. $350 \mathrm{~nm}$ excitation wavelength was used and emission spectra from $350 \mathrm{~nm}$ were collected with $0.65-\mathrm{nm}$ slit width for both. Raw data was normalized by subtracting baseline signal from initial incubation step. Sample was run in triplicate for each condition, then averaged and plotted with standard deviation in microsoft excel 16.51.

Negative stain Electron Microscopy. CTPS was assembled in reaction buffer (50mM TRIS or MES, $100 \mathrm{mM} \mathrm{NaCl}, 10 \mathrm{mM} \mathrm{MgCl} 2$ ) at $30^{\circ} \mathrm{C}$ for 15 minutes prior to applying $5 \mu$ l of sample to glow-discharged carbon-coated grids and incubated for 1 minute at room temperature. Grids were washed $3 x$ in $\mathrm{H}_{2} \mathrm{O}$ followed by $3 x$ in $0.7 \%$ uranyl formate with blotting in between all steps to remove excess liquid. Imaging was done on an FEI Morgagni electron microscope operating at an accelerating voltage of $100 \mathrm{kV}$. Datasets for hCTPS2 were collected on a Tecnai G2 Spirit (FEI) operating at $120 \mathrm{kV}$. Images were acquired at $\times 67,000$ magnification (pixel size $1.6 \AA / p x$ ) on a Ultrascan $40004 \mathrm{k} \times 4 \mathrm{k}$ CCD camera (Gatan). Contrast transfer function (CTF) was estimated using CTFFIND4 (Rohou and Grigorieff, 2015), Cryosparc v2.1 (Punjani et al., 2017) was used for automatic blob picking and 2D classification.

Cryo-electron microscopy sample preparation and data acquisition. Frozen URA7/URA8 was desalted into minimal buffer $(5 \mathrm{mM}$ MES $5.9,50 \mathrm{mM} \mathrm{NaCl})$ prior to assembly at $8 \mu \mathrm{M}$ with either substrates $(2 \mu \mathrm{M}$ UTP, $2 \mu \mathrm{M}$ ATP) or product $(2 \mu \mathrm{M}$ CTP). Reaction buffer contained $400 \mathrm{mM} \mathrm{NaCl}, 5 \mathrm{mM} \mathrm{MgCl} 2,3 \mathrm{mM}$ DTT, and 50mM MES either at pH 6.0 (predominantly 
bundles) or $\mathrm{pH} 6.5$ (predominantly single filaments). Protein was assembled for 15 minutes at $30^{\circ} \mathrm{C}$ then $3 \mathrm{ul}$ of the reaction material was double blotted (Snijder et al., 2017) onto glowdischarged C-FLAT 2/2 holey-carbon grids (Protochips), allowing a 1 minute room temperature incubation between sample depositions. Grids were subsequently blotted for 4.5 seconds using a Vitrobot MarkIV (ThermoFisher Scientific) with chamber conditions set to $100 \%$ humidity and at $4^{\circ} \mathrm{C}$. For Ura7 H360R sample was assembled at $5 \mathrm{uM}$ final concentration in $50 \mathrm{mM} \mathrm{NaCl}$, $50 \mathrm{mM}$ HEPES 7.5, and $10 \mathrm{mM} \mathrm{MgCl} 2$ then $3 \mathrm{ul}$ deposited onto lacey carbon grids which had thin carbon floated on top. Data were acquired using an FEI Titan Krios transmission electron microscope operating at 300kV and equipped with a Bioquantum GIF energy filter (Gatan) set to zero-loss mode with a slit width of $20 \mathrm{eV}$. Movies were collected on a K2 Summit Direct Detector camera (Gatan) in super-resolution mode at a magnification of $130 \mathrm{~K}$ (pixel size $0.525 \AA / p x$ ). Automatic data acquisition was done using the Leginon Software Package (Suloway et al., 2005) with a defocus range specified in table 1. Movies were acquired containing 50 frames having an exposure rate of $8.9 \mathrm{e}^{-} / \AA^{2} / \mathrm{sec}$ and a total dose of $89 \mathrm{e}^{-} / \AA^{2}$. For product-bound URA8, which exhibited a severe preferred orientation, we collected data with a stage tilt of both 20 and 40 degrees and combined the data for processing.

Cryo-EM data processing. Assembly conditions for filaments also contained some bundles, and vice versa, therefore these datasets were combined early on and bundles/filaments were separated through 2D classification and processed independently afterwards. See supplemental methods for further details. Briefly, images were manually curated to remove poor quality acquisitions such as bad ice or large regions of carbon. Dose-weighting and image alignment of all 50 frames was carried out using MotionCor2 (Zheng et al., 2017) with binning by a factor of 2 (final pixel size $1.05 \AA / p x$ ). Initial CTF parameters were estimated using GCTF (Zhang, 2016). Particle picking for bundles was done using the crYOLO (Wagner et al., 2019) helical pickier tool trained on both filaments and bundles and using a box size covering one tetramer, followed by manual picking to further improve the quality of the particle picks. Helical picking was chosen for the ease at the particle picking step, despite not processing any of the data with helical refinement. For tilted data we used the local GCTF per particle CTF estimation tool to improve per particle defocus values. To classify bundles from filaments, particles were extracted with a large box size (512 pixels). Particle stacks were exported to either cisTEM (Grant et al., 2018) or Cryosparc (Punjani et al., 2017) for iterative 2D reference-free classification. Starting models for all maps were always obtained ab initio, and for bundle data the process was repeated in cisTEM and later cryosparc as independent validation. All four bundle starting models were obtained in this way, and no symmetry was imposed. 3D classification of bundles in $\mathrm{C} 1$ yielded subsets differing in their arrangement of strands. Classes sharing a common core assembly pattern were combined for further processing. In all cases, the highest resolution bundle maps were obtained by $\mathrm{C} 2$ refinement of a masked central segment after performing signal subtraction of density outside this region. Early processing without imposing symmetry suggested overall two-fold symmetry in both bundle types. Therefore, we used a mask for focused alignment on 3-strands (staggered bundle; Ura7 product- and substrate-bound \& Ura8 substrate-bound) or 2 strands (in-register bundle; Ura8 product-bound) as these contained all the relevant contacts. FSC were calculated using Relion (Scheres, 2012) post-process or from the Phenix density modification (Terwilliger et al., 2020) output. Directional FSC was calculated using online FSC calculator (https://3dfsc.salk.edu/).

Atomic Model Building and Refinement. Initial models for Ura7 and Ura8 were obtained by threading their sequences onto the hCTPS1 substrate-bound structure from Lynch et al 2017. 
Where inserts existed models were built in manually using Coot (Emsley and Cowtan, 2004). Models for individual monomers were rigid body fit using Chimera (Pettersen et al., 2004) by domain (0-280 amido-ligase domain; 281-300 linker; 301-570 glutaminase domain) then backbone and side-chain positions refined with ISOLDE (Croll, 2018). C-termini were built manually in coot using the best available Ura7 map (product-bound) and Ura8 map (substratebound). ATP was modelled using ISOLDE, and UTP/CTP using Coot after all ISOLDE refinements. Ligands for product-bound Ura8 were rigid body fit in coot. The density corresponding to Ura8 residues 417-457 was found to be weaker and have relatively lower resolution than the rest of the structure. To build this loop the RosettaES (Frenz et al., 2017) loop modeling protocol in Rosetta (Alford et al., 2017) was used with a beamwidth of 256 . The top scoring result of the RosettaES protocol was selected and its geometries were refined with ISOLDE. Residues 444-455 were later removed due to poor angles and unsupported map density. After building the monomer, it was replicated at all symmetry equivalent positions to create the full tetramer, and a single monomer at the assembly interface. Residues at the tetramerization interface and assembly interface were relaxed using a full simulation in ISOLDE. Side chains and backbone angles were adjusted for a single monomer, which was again replicated to all four symmetry equivalent sites to generate the final tetramer model with identical subunits. Models were built into the high resolution product-bound Ura7 map, which was rigid body fit by domains (residues 0-273,276-299,302-C-ter) into lower resolution Ura7 product-bound bundle map based on local map quality. Junctions between domains were deleted (residues 274-275,300-301). For substrate-bound Ura7 filament, the Ura7-productbound filament model was rigid body fit by domain and junctions deleted (domains 0-280,281300,301-Cter; deleted 280-284 and 300-302). Clashes for this Ura7-substrate-bound model were removed using ISOLDE. Substrate-bound Ura7 bundle was built from a rigid body fit of the substrate-bound Ura7 filament model as described above. Ura8 bundle model building began with models from their corresponding ligand-state filament, rigid body fit by domain, then refined in ISOLDE. This monomer was duplicated at all relevant contact sites and overall relaxed in ISOLDE to refine positions at tetramerization interface, filament assembly interface, and lateral bundle contacts simultaneously. The monomer was then replicated at all symmetry sites and because not each monomer experiences the same lateral contacts, it was docked into different spots and an ISOLDE simulation was run with only the residues at the lateral interface with restraints on everything else. The final monomer was duplicated into all sites to fill the map density and ligands docked in and refined using coot. Model statistics were assessed using molprobity online server (http://molprobity.biochem.duke.edu/) and the RCSB PDB Submission validation report. Buried surface area for lateral bundle contacts and filament interface were calculated using PDBePISA tool (https://www.ebi.ac.uk/msd-srv/prot int/cgi-bin/piserver) with default parameters.

Substrate Tunnel Analysis. Caver 3.0.3 plugin (Chovancova et al., 2012) was used for Pymol 2.4.1 (The PyMOL Molecular Graphics System, n.d.) with default parameters. We set catalytic Cys404 as the start point for tunnel search of an individual monomer. Probe radius was set at $0.7 \AA$ and the tunnel from Cys 404 to the UTP base was identified. Inspection of radii along the length of the tunnel revealed that radii near the $\mathrm{P} 52 / \mathrm{C} 58 / \mathrm{H} 55$ constriction point in the Ura8 substrate-bound tetramer was between $1.2 \AA$ and $1.9 \AA$, consistent with relieved ammonia channel constriction (Lynch and Kollman, 2020).

Yeast Strain Construction and Media. Yeast were maintained in standard YPD (Lab Express) or synthetic media containing 2\% D-Glucose (Fisher). Background strains were W303 \{leu2- 
3,112 trp1-1 can1-100 ura3-1 ade2-1 his3-11,15\}. Deletion and mutation strains were made by a PCR approach as described previously (Wendland, 2003), and C-terminal tagging of yeast were made as described previously (Sheff and Thorn, 2004). List of yeast strains used can be found in Supplemental table 5.

Handling of Yeast Cells. To induce polymers, yeast were grown in YPD until mid-late log phase, washed in $1 \mathrm{X}$ PBS and resuspended in starvation media- 0.1M Phosphate-citrate buffer ( $\mathrm{pH} 5,6,7)$ and grown shaking at $30^{\circ} \mathrm{C}$ for 3-4 hours. To manipulate intracellular $\mathrm{pH}$ in the presence of $2 \%$ Glucose, $2 \mathrm{mM}$ of 2,4-Dinitrophenol (Sigma) was added to the media as described previously (Petrovska et al., 2014).

Yeast Growth Assays. Liquid growth curves were made by diluting mid-late log phase and/or starved yeast cells to OD600 0.05 in YPD or SD $+0.5 \%$ Glucose and growth was monitored on a Varioskan Lux plate reader at $600 \mathrm{~nm}$, shaking at $30{ }^{\circ} \mathrm{C}$ for 16 hours. Solid growth assays were done by making five 5-fold serial dilutions of mid-late log phase or staved yeast cells and plated on YPD or SD $+0.25 \%$ Glucose plates $+2 \%$ Agar and grown at 30 degrees for 48 hours.

Fluorescence Microscopy. Fixed and live fluorescence microscopy was done at $100 \mathrm{X}$ objective magnification on a DeltaVision Elite microscope (GE) equipped with DIC optics, using a $60 \times 1.42$ NA objective, and a sCMOS 5.4 PCle air-cooled camera (PCO-TECH).

Deconvolution was performed with SoftWorx (API, Issaquah, WA) and images were analyzed using Fiji, ImageJ (Schindelin et al., 2012). Figures were assembled using Adobe Photoshop. Representative images shown of experiments done on three independent replicates.

Fluorophores used were GFP and mCherry.

\section{Supplemental Movies:}

Movie 1. Ura8 conformational changes. Model morph from Ura8 product-bound filament to substrates-bound filament to substrate-bound tetramer. Ligands are shown at each step.

Movie 2. Wild type Ura7 polymerization. Yeast expressing wild type GFP-Ura7 upon transfer to starvation media. Scale bar is 5 microns.

Movie 3. Ura7-H360R polymerization. Yeast expressing GFP-Ura7-H360R upon transfer to starvation media. Scale bar is 5 microns.

Movie 4. Wild type Ura7 depolymerization. Yeast expressing wild type GFP-Ura7 after 4 hours of starvation then transferred to nutrient rich media. Scale bar is 5 microns.

Movie 5. Wild Ura7-H360R depolymerization. Yeast expressing GFP-Ura7-H360R after 4 hours of starvation then transferred to nutrient rich media. Scale bar is 5 microns.

Movie 6. Masked cryo-EM density of substrate-bound Ura8 bundle. Final reconstruction (C2 symmetry imposed) of a 3 stranded bundle segment. Each strand is either blue or orange, 
with protomers alternating shade. A left/right half a tetramer is in the central reconstruction (orange), with two filament assembly interfaces on either side (blue).

Movie 7. Atomic model of substrate-bound Ura8 bundle. Surface rendering of models for 3 strands built into the final reconstruction, which shows the full scale of the bundle. Each strand is in either orange or blue with protomers shaded differently.

Movie 8. Masked cryo-EM density of product-bound Ura8 bundle. Final reconstruction (C2 symmetry imposed) of a 2 stranded bundle segment. Each strand is either blue or orange, with protomers alternating shade.

Movie 9. Atomic model of product-bound Ura8 bundle. Surface rendering of models for 2 strands built into the final reconstruction, which shows the full scale of the bundle. Each strand is in either orange or blue with protomers shaded differently.

\section{References}

Alford RF, Leaver-Fay A, Jeliazkov JR, O’Meara MJ, DiMaio FP, Park H, Shapovalov MV, Renfrew PD, Mulligan VK, Kappel K, Labonte JW, Pacella MS, Bonneau R, Bradley P, Dunbrack RL, Das R, Baker D, Kuhlman B, Kortemme T, Gray JJ. 2017. The Rosetta All-Atom Energy Function for Macromolecular Modeling and Design. J Chem Theory Comput 13:3031-3048. doi:10.1021/acs.jctc.7b00125

Barry RM, Bitbol A-F, Lorestani A, Charles EJ, Habrian CH, Hansen JM, Li H-J, Baldwin EP, Wingreen NS, Kollman JM, Gitai Z. 2014. Large-scale filament formation inhibits the activity of CTP synthetase. eLife 3:e03638. doi:10.7554/eLife.03638

Carcamo WC, Satoh M, Kasahara H, Terada N, Hamazaki T, Chan JYF, Yao B, Tamayo S, Covini G, von Mühlen CA, Chan EKL. 2011. Induction of Cytoplasmic Rods and Rings Structures by Inhibition of the CTP and GTP Synthetic Pathway in Mammalian Cells. PLOS ONE 6:e29690. doi:10.1371/journal.pone.0029690

Chang Y-F, Carman GM. 2008. CTP synthetase and its role in phospholipid synthesis in the yeast Saccharomyces cerevisiae. Progress in Lipid Research 47:333-339. doi:10.1016/j.plipres.2008.03.004

Choi M-G, Park T-S, Carman GM. 2003. Phosphorylation of Saccharomyces cerevisiae CTP Synthetase at Ser424 by Protein Kinases A and C Regulates Phosphatidylcholine Synthesis by the CDP-choline Pathway. Journal of Biological Chemistry 278:2361023616. doi:10.1074/jbc.M303337200

Chovancova E, Pavelka A, Benes P, Strnad O, Brezovsky J, Kozlikova B, Gora A, Sustr V, Klvana M, Medek P, Biedermannova L, Sochor J, Damborsky J. 2012. CAVER 3.0: A Tool for the Analysis of Transport Pathways in Dynamic Protein Structures. PLoS Comput Biol 8:e1002708. doi:10.1371/journal.pcbi.1002708

Cohen RJ, Judith A J, George B. B. 1976. The functional relationship between polymerization and catalytic activity of beef liver glutamate dehydrogenase: II. jmb 108:179-199. doi:https://doi.org/10.1016/S0022-2836(76)80102-7

Croll TI. 2018. ISOLDE: a physically realistic environment for model building into low-resolution electron-density maps. Acta Cryst D 74:519-530. doi:10.1107/S2059798318002425

Emsley P, Cowtan K. 2004. Coot : model-building tools for molecular graphics. Acta Crystallogr D Biol Crystallogr 60:2126-2132. doi:10.1107/S0907444904019158

Endrizzi JA, Kim H, Anderson PM, Baldwin EP. 2005. Mechanisms of Product Feedback 
Regulation and Drug Resistance in Cytidine Triphosphate Synthetases from the Structure of a CTP-Inhibited Complex '. Biochemistry 44:13491-13499. doi:10.1021/bi051282o

Endrizzi JA, Kim H, Anderson PM, Baldwin EP. 2004. Crystal Structure of Escherichia coli Cytidine Triphosphate Synthetase, a Nucleotide-Regulated Glutamine Amidotransferase/ATP-Dependent Amidoligase Fusion Protein and Homologue of Anticancer and Antiparasitic Drug Targets ${ }^{\dagger}{ }^{\ddagger}$. Biochemistry 43:6447-6463. doi:10.1021/bi0496945

Fairbanks LD, Bofill M, Ruckemann K, Simmonds HA. 1995. Importance of Ribonucleotide Availability to Proliferating T-lymphocytes from Healthy Humans. Journal of Biological Chemistry 270:29682-29689. doi:10.1074/jbc.270.50.29682

Frenz B, Walls AC, Egelman EH, Veesler D, DiMaio F. 2017. RosettaES: a sampling strategy enabling automated interpretation of difficult cryo-EM maps. Nat Methods 14:797-800. doi:10.1038/nmeth.4340

Frey TG, Eisenberg D, Eiserling FA. 1975. Glutamine synthetase forms three- and sevenstranded helical cables. Proc Natl Acad Sci U S A 72:3402-3406.

Garcia-Seisdedos H, Empereur-Mot C, Elad N, Levy ED. 2017. Proteins evolve on the edge of supramolecular self-assembly. Nature 548:244-247. doi:10.1038/nature23320

Goto M, Omi R, Nakagawa N, Miyahara I, Hirotsu K. 2004. Crystal Structures of CTP Synthetase Reveal ATP, UTP, and Glutamine Binding Sites. Structure 12:1413-1423. doi:10.1016/j.str.2004.05.013

Grant T, Rohou A, Grigorieff N. 2018. cisTEM, user-friendly software for single-particle image processing. eLife 7:e35383. doi:10.7554/eLife.35383

Habrian C, Chandrasekhara A, Shahrvini B, Hua B, Lee J, Jesinghaus R, Barry R, Gitai Z, Kollman J, Baldwin EP. 2016. Inhibition of Escherichia coli CTP Synthetase by NADH and Other Nicotinamides and Their Mutual Interactions with CTP and GTP. Biochemistry 55:5554-5565. doi:10.1021/acs.biochem.6b00383

Hunkeler M, Hagmann A, Stuttfeld E, Chami M, Guri Y, Stahlberg H, Maier T. 2018. Structural basis for regulation of human acetyl-CoA carboxylase. Nature 558:470-474. doi:10.1038/s41586-018-0201-4

Ingerson-Mahar M, Briegel A, Werner JN, Jensen GJ, Gitai Z. 2010. The metabolic enzyme CTP synthase forms cytoskeletal filaments. Nat Cell Biol 12:739-746. doi: $10.1038 /$ ncb2087

Johnson MC, Kollman JM. 2020. Cryo-EM structures demonstrate human IMPDH2 filament assembly tunes allosteric regulation. eLife 9:e53243. doi:10.7554/eLife.53243

Kemp RG. 1971. Rabbit Liver Phosphofructokinase. Journal of Biological Chemistry 246:245252. doi:10.1016/S0021-9258(18)62556-6

Kleinschmidt AK, Moss J, Lane D. 1969. Acetyl coenzyme A carboxylase: filamentous nature of the animal enzymes. Science 166:1276-1278.

Lieberman I. 1956. ENZYMATIC AMINATION OF URIDINE TRIPHOSPHATE TO CYTIDINE TRIPHOSPHATE. Journal of Biological Chemistry 222:765-775. doi:10.1016/S00219258(20)89934-7

Liu H, Naismith JH. 2008. An efficient one-step site-directed deletion, insertion, single and multiple-site plasmid mutagenesis protocol. BMC Biotechnol 8:91. doi:10.1186/14726750-8-91

Liu J-L. 2010. Intracellular compartmentation of CTP synthase in Drosophila. Journal of Genetics and Genomics 37:281-296. doi:10.1016/S1673-8527(09)60046-1

Lynch EM, Hicks DR, Shepherd M, Endrizzi JA, Maker A, Hansen JM, Barry RM, Gitai Z, Baldwin EP, Kollman JM. 2017. Human CTP synthase filament structure reveals the active enzyme conformation. Nat Struct Mol Biol 24:507-514. doi:10.1038/nsmb.3407

Lynch EM, Kollman JM. 2020. Coupled structural transitions enable highly cooperative 
regulation of human CTPS2 filaments. Nat Struct Mol Biol 27:42-48.

doi:10.1038/s41594-019-0352-5

Lynch EM, Kollman JM, Webb BA. 2020. Filament formation by metabolic enzymes-A new twist on regulation. Current Opinion in Cell Biology 66:28-33.

doi:10.1016/j.ceb.2020.04.006

Miller RE, Shelton E, Stadtman ER. 1974. Zinc-induced paracrystalline aggregation of glutamine synthetase. Archives of Biochemistry and Biophysics 163:155-171. doi:10.1016/00039861(74)90465-2

Nadkarni AK, McDonough VM, Yang W-L, Stukey JE, Ozier-Kalogeropoulos O, Carman GM. 1995. Differential Biochemical Regulation of the URA7- and URA8-encoded CTP Synthetases from Saccharomyces cerevisiae. Journal of Biological Chemistry 270:24982-24988. doi:10.1074/jbc.270.42.24982

Narayanaswamy R, Levy M, Tsechansky M, Stovall GM, O’Connell JD, Mirrielees J, Ellington AD, Marcotte EM. 2009. Widespread reorganization of metabolic enzymes into reversible assemblies upon nutrient starvation. PNAS 106:10147-10152. doi:10.1073/pnas.0812771106

Noree C, Begovich K, Samilo D, Broyer R, Monfort E, Wilhelm JE. 2019. A quantitative screen for metabolic enzyme structures reveals patterns of assembly across the yeast metabolic network. MBoC 30:2721-2736. doi:10.1091/mbc.E19-04-0224

Noree C, Sato BK, Broyer RM, Wilhelm JE. 2010. Identification of novel filament-forming proteins in Saccharomyces cerevisiae and Drosophila melanogaster. Journal of Cell Biology 190:541-551. doi:10.1083/jcb.201003001

Orij R, Postmus J, Ter Beek A, Brul S, Smits GJ. 2009. In vivo measurement of cytosolic and mitochondrial $\mathrm{pH}$ using a $\mathrm{pH}$-sensitive GFP derivative in Saccharomyces cerevisiae reveals a relation between intracellular pH and growth. Microbiology 155:268-278. doi:10.1099/mic.0.022038-0

Ozier-Kalogeropoulos O, Adeline M-T, Yang W-L, Carman GM, Lacroute F. 1994. Use of synthetic lethal mutants to clone and characterize a novel CTP synthetase gene in Saccharomyces cerevisiae. Molec Gen Genet 242:431-439. doi:10.1007/BF00281793

Park CK, Horton NC. 2019. Structures, functions, and mechanisms of filament forming enzymes: a renaissance of enzyme filamentation. Biophys Rev 11:927-994. doi:10.1007/s12551-019-00602-6

Park T-S, O'Brien DJ, Carman GM. 2003. Phosphorylation of CTP Synthetase on Ser36, Ser330, Ser354, and Ser454 Regulates the Levels of CTP and Phosphatidylcholine Synthesis in Saccharomyces cerevisiae. Journal of Biological Chemistry 278:2078520794. doi:10.1074/jbc.M301394200

Park T-S, Ostrander DB, Pappas A, Carman GM. 1999. Identification of Ser ${ }^{424}$ as the Protein Kinase A Phosphorylation Site in CTP Synthetase from Saccharomyces cerevisiae. Biochemistry 38:8839-8848. doi:10.1021/bi990784x

Petrovska I, Nüske E, Munder MC, Kulasegaran G, Malinovska L, Kroschwald S, Richter D, Fahmy K, Gibson K, Verbavatz J-M, Alberti S. 2014. Filament formation by metabolic enzymes is a specific adaptation to an advanced state of cellular starvation. eLife 3:e02409. doi:10.7554/eLife.02409

Pettersen EF, Goddard TD, Huang CC, Couch GS, Greenblatt DM, Meng EC, Ferrin TE. 2004. UCSF Chimera?A visualization system for exploratory research and analysis. J Comput Chem 25:1605-1612. doi:10.1002/jcc.20084

Punjani A, Rubinstein JL, Fleet DJ, Brubaker MA. 2017. cryoSPARC: algorithms for rapid unsupervised cryo-EM structure determination. Nat Methods 14:290-296. doi:10.1038/nmeth.4169

Rohou A, Grigorieff N. 2015. CTFFIND4: Fast and accurate defocus estimation from electron micrographs. Journal of Structural Biology, Recent Advances in Detector Technologies 
and Applications for Molecular TEM 192:216-221. doi:10.1016/j.jsb.2015.08.008

Scheres SHW. 2012. RELION: Implementation of a Bayesian approach to cryo-EM structure determination. J Struct Biol 180:519-530. doi:10.1016/j.jsb.2012.09.006

Schmidt TT, Reyes G, Gries K, Ceylan CÜ, Sharma S, Meurer M, Knop M, Chabes A, Hombauer H. 2017. Alterations in cellular metabolism triggered by URA7 or GLN3 inactivation cause imbalanced dNTP pools and increased mutagenesis. Proc Natl Acad Sci USA 114:E4442-E4451. doi:10.1073/pnas.1618714114

Sheff MA, Thorn KS. 2004. Optimized cassettes for fluorescent protein tagging in Saccharomyces cerevisiae. Yeast 21:661-670. doi:10.1002/yea.1130

Shen Q-J, Kassim H, Huang Y, Li H, Zhang J, Li G, Wang P-Y, Yan J, Ye F, Liu J-L. 2016. Filamentation of Metabolic Enzymes in Saccharomyces cerevisiae. Journal of Genetics and Genomics 43:393-404. doi:10.1016/j.jgg.2016.03.008

Simonet JC, Burrell AL, Kollman JM, Peterson JR. 2020. Freedom of assembly: metabolic enzymes come together. Mol Biol Cell 31:1201-1205. doi:10.1091/mbc.E18-10-0675

Snijder J, Borst AJ, Dosey A, Walls AC, Burrell A, Reddy VS, Kollman JM, Veesler D. 2017. Vitrification after multiple rounds of sample application and blotting improves particle density on cryo-electron microscopy grids. J Struct Biol 198:38-42. doi:10.1016/j.jsb.2017.02.008

Stoddard PR, Lynch EM, Farrell DP, Dosey AM, DiMaio F, Williams TA, Kollman JM, Murray AW, Garner EC. 2020. Polymerization in the actin ATPase clan regulates hexokinase activity in yeast. Science 367:1039-1042. doi:10.1126/science.aay5359

Suloway C, Pulokas J, Fellmann D, Cheng A, Guerra F, Quispe J, Stagg S, Potter CS, Carragher B. 2005. Automated molecular microscopy: the new Leginon system. J Struct Biol 151:41-60. doi:10.1016/j.jsb.2005.03.010

Terwilliger TC, Ludtke SJ, Read RJ, Adams PD, Afonine PV. 2020. Improvement of cryo-EM maps by density modification. Nat Methods 17:923-927. doi:10.1038/s41592-020-09149

The PyMOL Molecular Graphics System. n.d. . Schrödinger, LLC.

Trotta PP, Wellner VP, Pinkus LM, Meister A. 1973. Observations on the pH Dependence of the Glutaminase Activity of a Glutamine Amidotransferase, Carbamylphosphate Synthetase. Proceedings of the National Academy of Sciences 70:2717-2721. doi:10.1073/pnas.70.10.2717

Wagner T, Merino F, Stabrin M, Moriya T, Antoni C, Apelbaum A, Hagel P, Sitsel O, Raisch T, Prumbaum D, Quentin D, Roderer D, Tacke S, Siebolds B, Schubert E, Shaikh TR, Lill P, Gatsogiannis C, Raunser S. 2019. SPHIRE-crYOLO is a fast and accurate fully automated particle picker for cryo-EM. Commun Biol 2:1-13. doi:10.1038/s42003-0190437-z

Webb BA, Dosey AM, Wittmann T, Kollman JM, Barber DL. 2017. The glycolytic enzyme phosphofructokinase-1 assembles into filaments. Journal of Cell Biology 216:23052313. doi:10.1083/jcb.201701084

Wendland J. 2003. PCR-based methods facilitate targeted gene manipulations and cloning procedures. Curr Genet 44:115-123. doi:10.1007/s00294-003-0436-x

Yang W-L, McDonough VM, Ozier-Kalogeropoulos O, Adeline M-T, Flocco MT, Carman GM. 1994. Purification and Characterization of CTP Synthetase, the Product of the URA7 Gene in Saccharomyces cerevisiae. Biochemistry 33:10785-10793. doi:10.1021/bi00201a028

Zhang B, Zhang Y, Liu J-L. 2021. Highly effective proximate labeling in Drosophila. G3 Genes|Genomes|Genetics 11. doi:10.1093/g3journal/jkab077

Zhang K. 2016. Gctf: Real-time CTF determination and correction. Journal of Structural Biology 193:1-12. doi:10.1016/j.jsb.2015.11.003

Zhang S, Ding K, Shen Q-J, Zhao S, Liu J-L. 2018. Filamentation of asparagine synthetase in 
Saccharomyces cerevisiae. PLoS Genet 14:e1007737. doi:10.1371/journal.pgen.1007737

Zheng SQ, Palovcak E, Armache J-P, Verba KA, Cheng Y, Agard DA. 2017. MotionCor2: anisotropic correction of beam-induced motion for improved cryo-electron microscopy. Nat Methods 14:331-332. doi:10.1038/nmeth.4193

Zhou X, Guo C-J, Chang C-C, Zhong J, Hu H-H, Lu G-M, Liu J-L. 2021. Structural basis for ligand binding modes of CTP synthase. PNAS 118. doi:10.1073/pnas.2026621118

Zhou X, Guo C-J, Hu H-H, Zhong J, Sun Q, Liu D, Zhou S, Chang CC, Liu J-L. 2019. Drosophila CTP synthase can form distinct substrate- and product-bound filaments. Journal of Genetics and Genomics 46:537-545. doi:10.1016/j.jgg.2019.11.006

\section{Acknowledgements}

The authors thank the Arnold and Mabel Beckman CryoEM Center at the University of Washington for electron microscope use. We thank Kelli L. Hvorecny, Anika L. Burrell, and John Calise for valuable feedback, especially Anika L. Burrell who provided generous feedback to significantly improve the manuscript. This work was funded by the US National Institutes of Health (R01 GM118396, S10 OD032290, to J.M.K. and T32 GM007270 to J.M.H.). J.M.H. thanks Kim Hansen for support and valuable discussion, and Murphy Hansen for tools aiding in structure determination.

\section{Contributions}

J.M.H. performed structural, biochemical, biophysical assays, and accompanying data analysis. A.H. performed yeast manipulations, fluorescence imaging, growth assays, and accompanying data analysis. J.M.H., J.M.K., A.H., and E.M.L. designed experiments. J.Q. optimized cryoEM sample preparation. D.P.F. performed mutagenesis. D.P.F. and F.D. built a segment of model with Rosetta. J.M.H. and J.M.K. wrote the manuscript.

\section{Competing Interests}

No competing interests declared. 